Louisiana State University

LSU Digital Commons

1981

\title{
Electron-Phonon Scattering Rates in Antimony: Radio-Frequency Size Effect.
}

Thomas Clifton O'hara

Louisiana State University and Agricultural \& Mechanical College

Follow this and additional works at: https://digitalcommons.Isu.edu/gradschool_disstheses

\section{Recommended Citation}

O'hara, Thomas Clifton, "Electron-Phonon Scattering Rates in Antimony: Radio-Frequency Size Effect." (1981). LSU Historical Dissertations and Theses. 3649.

https://digitalcommons.Isu.edu/gradschool_disstheses/3649

This Dissertation is brought to you for free and open access by the Graduate School at LSU Digital Commons. It has been accepted for inclusion in LSU Historical Dissertations and Theses by an authorized administrator of LSU Digital Commons. For more information, please contact gradetd@lsu.edu. 


\section{INFORMATION TO USERS}

This was produced from a copy of a document sent to us for microfilming. While the most advanced technological means to photograph and reproduce this document have been used, the quality is heavily dependent upon the quality of the material submitted.

The following explanation of techniques is provided to help you understand markings or notations which may appear on this reproduction.

1. The sign or "target" for pages apparently lacking from the document photographed is "Missing Page(s)". If it was possible to obtain the missing page(s) or section, they are spliced into the film along with adjacent pages. This may have necessitated cutting through an image and duplicating adjacent pages to assure you of complete continuity.

2. When an image on the film is obliterated with a round black mark it is an indication that the film inspector noticed either blurred copy because of movement during exposure, or duplicate copy. Unless we meant to delete copyrighted materials that should not have been filmed, you will find a good image of the page in the adjacent frame. If copyrighted materials were deleted you will find a target note listing the pages in the adjacent frame.

3. When a map, drawing or chart, etc., is part of the material being photographed the photographer has followed a definite method in "sectioning" the material. It is customary to begin filming at the upper left hand corner of a large sheet and to continue from left to right in equal sections with small overlaps. If necessary, sectioning is continued again-beginning below the first row and continuing on until complete.

4. For any illustrations that cannot be reproduced satisfactorily by xerography, photographic prints can be purchased at additional cost and tipped into your xerographic copy. Requests can be made to our Dissertations Customer Services Department.

5. Some pages in any document may have indistinct print. In all cases we have filmed the best available copy.

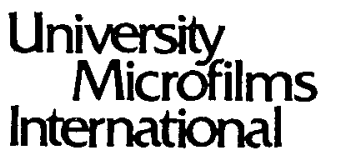


O'HaRa, THOMAS CLIfTON

ELECTRON-PHONON SCATTERING RATES IN ANTIMONY: RADIOFREQUENCY SIZE EFFECT

The Louisiana State University and Agricultural and Mechanical Col. PH.D. 1981

\author{
University \\ Microfilms \\ International 300 N. Zeeb Road, An Arbor, MI 483106
}


In all cases this material has been filmed in the best possible way from the available copy.

Problems encountered with this document have been identified here with a check mark

1. Glossy photographs or pages

2. Colored illustrations, paper or print

3. Photographs with dark background

4. Illustrations are poor copy

5. Pages with black marks, not original copy

6. Print shows through as there is text on both sides of page

7. Indistinct, broken or small print on several pages

8. Print exceeds margin requirements

9. Tightly bound copy with print lost in spine

10. Computer printout pages with indistinct print

11. Page(s)____ lacking when material received, and not available from school or author.

12. Page(s) seem to be missing in numbering only as text follows.

13. Two pages numbered Text follows.

14. Curling and wrinkled pages

15. Other

\section{University Microfilms International}


ELECTRON-PHONON SCATTERING

RATES IN ANTIMONY:

RADIO-FREQUENCY STZE EFFECT

\section{A Dissertation}

Submitted to the Graduate Faculty of the Louisiana state University and Agricultural and Mechanical College in partial fulfillment of the requirements for the degree of Doctor of Philosophy

in

The Department of Physics and Astronomy

by

Thomas Clifton O'Hara

B.S., The University of Texas, 1969

M.S., Louisiana State University, 1973

August 1981 
The author wishes foremost to express a sincere appreciation to Dr. Roy G. Goodrich for suggesting the problem and providing guidance and assistance throughout the course of the experiment. Special gratitude is extended to Mr. Phillip R. Broussard for his assistance with the computer programming. Thanks are due to Drs. John C. Kimball and claude G. Grenier for many informative discussions.

The author wishes to express thanks to the staffs of the machine and electronics shops, in particular, Mr. Harold Bennett, for their help in preparation of the experiment. Special thanks are due to the author's wife, Joy O'Hara, for being patient and supportive throughout the progress of the experiment.

The author wishes to express his appreciation to the Dr. Charles E. Coates Memorial Fund of the LsU Foundation for partial financial assistance in the preparation of this manuscript. 


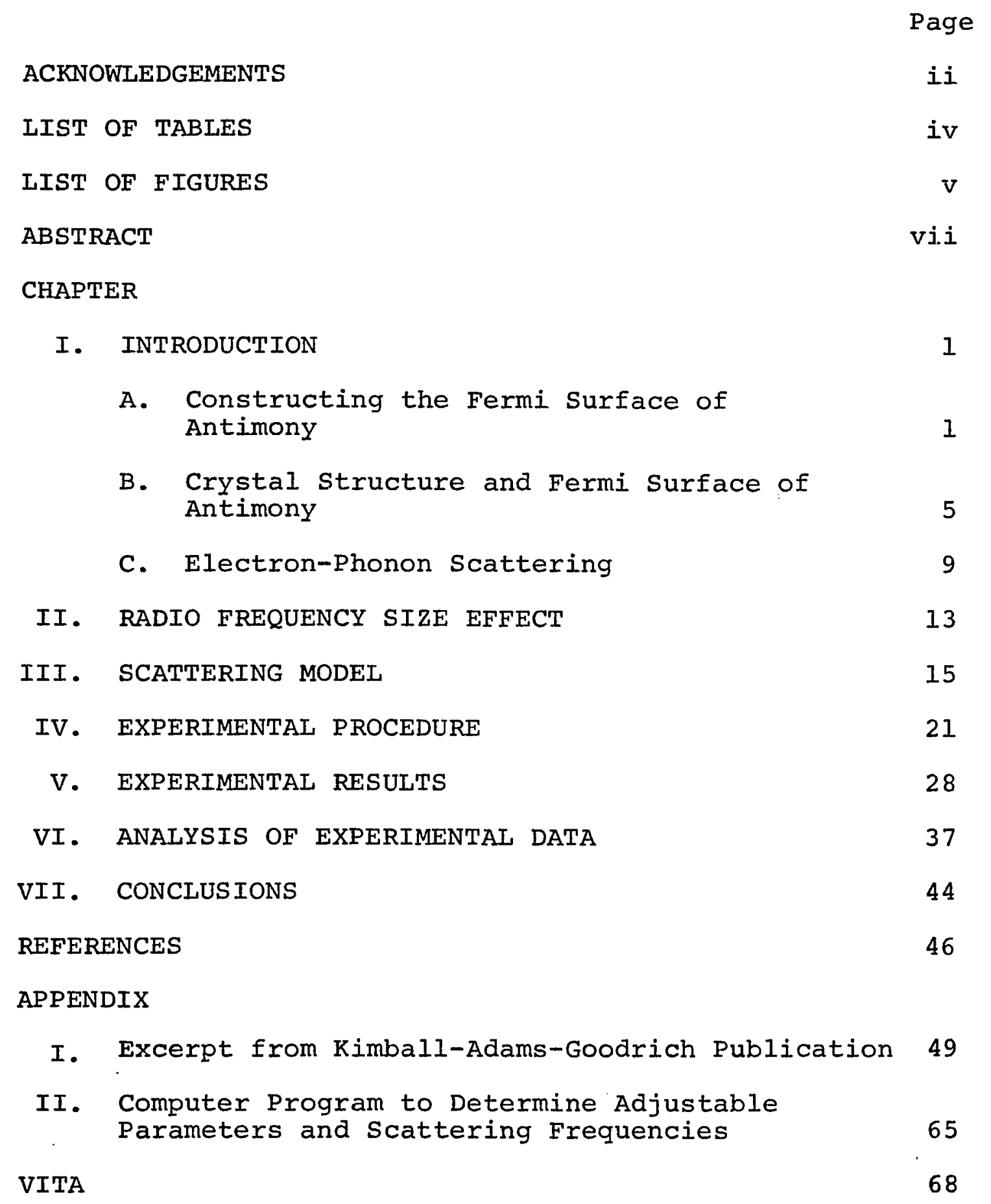




\section{LIST OF TABLES}

Table

Page

I. Crystal structure parameters for antimony at 4. $2 \mathrm{~K}$.

II. Measured slopes of $\ln \left(A / A_{0}\right)$ Vs. $T^{2}$ and effective masses for antimony orbits.

III. Measured slopes for orbits in antimony at three thicknesses.

IV. Electron-phonon and impurity scattering rates for orbits in the bisectrix plane on the hole surface of antimony.

V. Electron-phonon scattering rates for electron orbits in the bisectrix plane of antimony.

VI. Electron-phonon scattering rates for the end on electron surface in the bisectrix plane. 
LIST OF FIGURES

Figure

Page

1. The Brij.louin zone for antimony.

2. The Brillouin zone of antimony and the rhombohedron of twice the volume from which it is obtained. In addition are shown: the trigonal and binary axes; the rectangular $x$, y. z coordinate system; and the reciprocal lattice vectors $\underline{g_{1}}, \underline{g_{2}}, \underline{g_{3}}$ of the A7 structure. 8

3. Schematic diagram of experimental apparatus.

4. Double-walled can arrangement containing the sample in the experimental apparatus.

5. Typical set of raw RFSE data.

6. The Fermi surface of antimony within the Brillouin zone.

7. Projections of pieces of the electron and hole FS of antimony onto the bisectrix plane. The open circles indicate the orbits on the hole surface where data were obtained and the solid circles are for orbits on the two pieces of the electron surface.

8. Sample of data which displayed anomalous behavior as a function of temperature. Over part of the temperature range, the amplitude increased with temperature. 


\section{LIST OF FIGURES (continued)}

Figure

Page

9. Diagrams for scattering processes considered in deriving Eq. (2). The vectors $\vec{k}$ represents electrons contributing to a signal, thus $a$ and c represent scattering out of an orbit and b and $d$ into the orbit.

10. The current and sample configuration which lead to a $\mathrm{T}^{5}$ dependence of $\ln \mathrm{A}$.

11. Geometry necessary for the scattering effectiveness calculation.

12. Results of numerical integration for $\gamma(q)$ and the approximate function used for $\gamma(q)$ in the fitting procedure. 


\begin{abstract}
Temperature dependent radio-frequency size effect measurements have been performed on high-purity, single crystal antimony plates at thicknesses of $0.592 \mathrm{~mm}$, $0.347 \mathrm{~mm}$, and $0.238 \mathrm{~mm}$. The natural logs of the amplitudes of the data are found experimentally proportional to $\mathrm{T}^{2}$ in agreement with previous measurements. However, the data are analyzed according to a model in which the electronphonon scattering frequencies are proportional to $T^{3}$, with the explanation that the $\mathrm{T}^{2}$ behavior is due to the small pieces of Fermi surface in antimony. The electron-phonon scattering frequencies are determined for both holes and electrons and are found to be highly anisotropic in each case. A copy of the computer program used in the data analysis is included in Appendix II.
\end{abstract}

vij 
CHAPTER I

\section{INTRODUCTION}

A. Constructing the Fermi Surface of Antimony

Over the last thirty years a number of experimental techniques have evolved for studying the electronic structure of metals. One particular result of these investigations has been a bettex understanding of the Fermi surface, a constant energy surface in the metal which, at a temperature of $0 \mathrm{k}$, separates the occupied from the unoccupied electron states in $\mathrm{k}$-space. The four most informative techniques will be summarized here.

In the de Haas-van Alphen effect, the experimenter places the metal in an increasing magnetic field; and, as the quantized energy states in the metal pass through the Fermi level, he measures the influence of the states on the magnetic susceptibility or the transport properties (de Haas-Shubnikov effect, for example) of the metal. The extremal areas of the various pieces of the Fermi surface are deduced from the periodic variation of these properties as the field is increased. Unfortunately, only the area and not the shape of a particular section of the Fermi surface can be obtained.

In cyclotron resonance experiments, the rate of change of area with respect to energy can be found at the Fermi surface. Theoretically, the Fermi surface can be 
constructed once its area and the derivative of the area with respect to energy are known. In practice this is a very difficult procedure requiring the fitting of data to assumed models. In addition the samples are very difficult to prepare properly, and the applied radiation frequency must be much greater than the carrier scattering frequency for resonance (and signals) to occur.

There are two more direct methods available for calipering across extremal dimensions of the Fermi surface and thereby finding its size and shape. The first of these involves the use of ultrasonic attenuation. Ultrasound is propagated in a sample with the sound wave perpendicular to an increasing magnetic field. Oscillatory changes in the attenuation of the ultrasound occur when diameters of extremal orbits of carriers on the Fermi surface become equal to multiples of the ultrasound wavelength. This may be observed when the mean free path of the carriers is long compared to the acoustic wavelength and the gross acoustic attenuation is small so that the small oscillatory changes are not masked.

The other method for studying extremal dimensions is the radio-frequency size effect (RFSE). The rf surface impedance of a thin, single crystal plate is studied as a function of a magnetic field applied in the plane of the plate. When an extremal electronic orbit on the Fermi surface exactly spans the thickness of the metal plate, the 
surface impedance exhibits discontinuities. The extremal dimension of the Fermi surface is directly proportional to the magnetic field at which this discontinuity occurs. This technique does require very pure samples in which the mean free path is several times the sample thickness. The RFSE will be discussed more completely in Chapter II of this dissertation.

The FS of antimony has been studied by each of the above methods. The investigators have included Eckstein ${ }^{1}$ (ultrasonic attenuation); Ericksson, Beckman, and Hornfeldt ${ }^{2}$ (ultrasonic attenuation); Ketterson ${ }^{3}$ (de Haasvan Alphen); Korolyuk and Matsakov ${ }^{4}$ (geometric resonance); Rao, et al. ${ }^{5}$ (de Haas-Shubnikov); Lerner and Eastman ${ }^{6}$ (de Haas-Shubnikov); Datars and Vanderkooy ${ }^{7}$ (cyclotron resonance); and Windmiller ${ }^{8}$ (de Haas-van Alphen).

The interpretation of these experiments was confusing until Falicov and $\operatorname{Lin}^{9}$ (FL) performed an energy band calculation. Via this calculation, they demonstrated that antimony has three electron pockets located at the $I$ point in the Brillouin zone (BZ) and six hole pockets in the mirror plane near the $\mathrm{T}$ point in the $\mathrm{BZ}$ (Fig. 1). While this calculation resolved the situation concerning the sign of the carriers, number of pockets of each, and their location in the $\mathrm{BZ}$, it did not predict the shapes of these pieces of the FS.

Herrod, Gage, and Goodrich ${ }^{10}$ used RFSE to produce a detailed picture of the FS of antimony. In addition they 


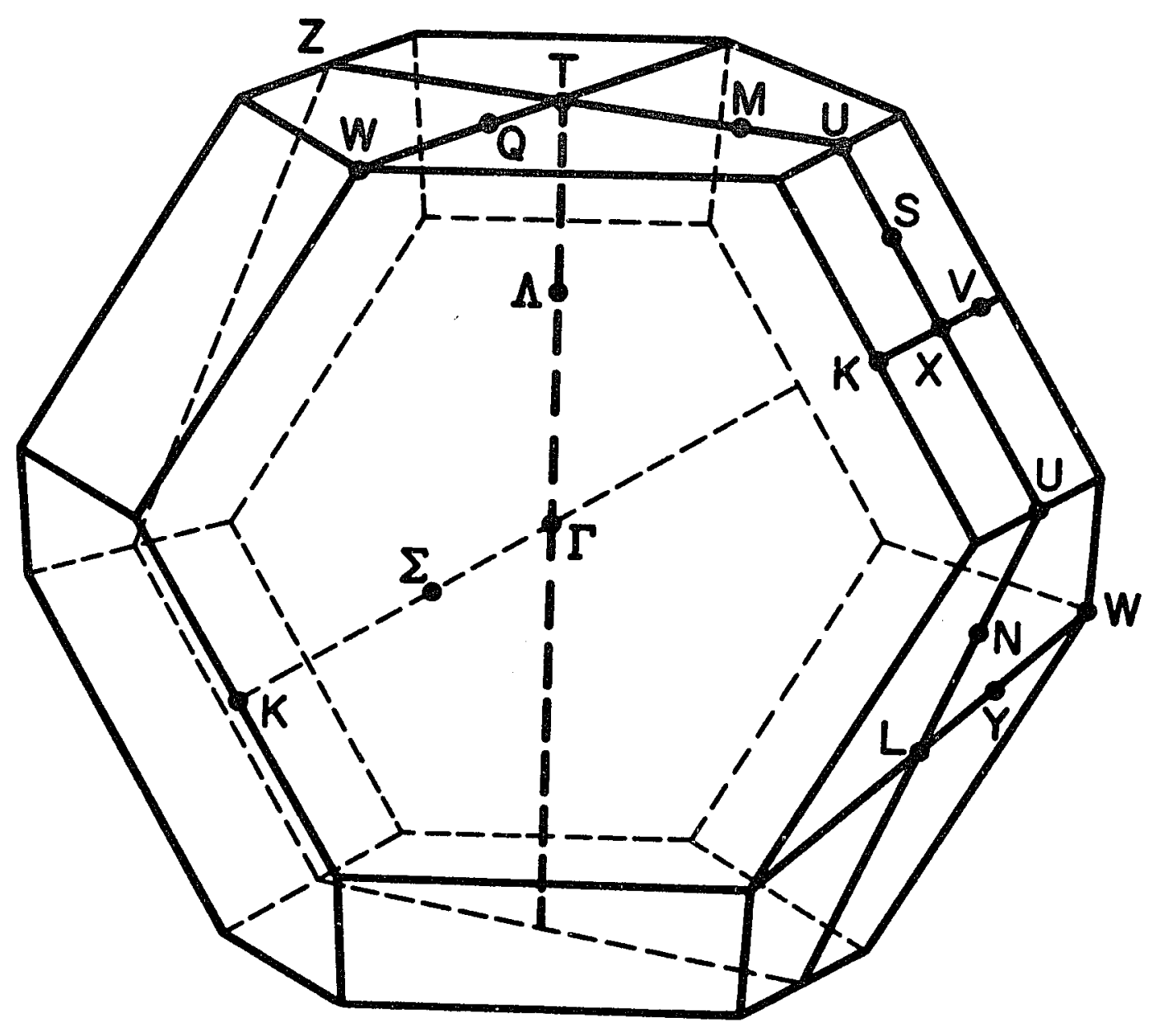

Fig. 1 
used this data in combination with the pseudopotential calculation of FI to yield a modified picture of the band structure.

Suematsu, Koshino, and Tanuma ${ }^{11}$ used microwave resonance in order to study magnetic surface states in antimony. In the process, they again recorded the fact which had been shown in previous experimental measurements and band calculations that the Fermi surfaces are not precisely ellipsoidal but deviate from ellipsoids in a complicated fashion.

\section{B. Crystal Structure and Fermi surface of Antimony}

Antimony is a group-V semimetal (atomic number 51) which has the A7 (arsenic) crystal structure. The unit cell (which is also a primitive cell) is rhombohedral and contains two atoms.

The structure can be obtained from a simple cubic structure by making slight displacements of the atoms. Initially, let us separate a simple cubic lattice into two face-centered sublattices where the two sublattices are related by a translation along the body-diagonal of the original simple cube. The parameter u is used to characterize the displacement. If $2 \underline{t}$ is the smaller vector in the trigonal direction separating the two interpenetrating lattices, and $\underline{d}$ is the trigonal body-diagonal of one of the lattices, then 


$$
\underline{t}=\underline{u d}, \quad u \leq 1 / 4
$$

The value $u=1 / 4$ corresponds to a simple cubic structure. Then by making a slight trigonal distortion (shear), we arrive at the structure of antimony. The extent of this distortion is small: $\alpha$, the rhombohedral angle between the primitive translations of the $A 7$ structure is $57^{\circ} 6^{\prime}$ for antimony as compared to $60^{\circ}$ for the undistorted simple cubic lattice.

In the presence of shear, the value $u=1 / 4$ now corresponds to a simple rhombohedral structure.

Table $I$ is taken from Herrod ${ }^{12}$ and contains a list of all the relevant crystal structure parameters of antimony at $4.2 \mathrm{~K}$. Figure 2 shows the Brillouin zone of antimony as well as the rhombohedron of twice the volume from which it is obtained. The axes labeled $x, y, z$ are the cubic axes in the absence of shear, and it is convenient to keep them as an orthogonal system of coordinates.

The three primitive translation vectors of the antimony lattice are then expressed as $a_{1}=a_{0}(\varepsilon, 1,1)$, $\underline{a_{2}}=a_{0}(1, \varepsilon, 1)$, and $\underline{a_{3}}=a_{0}(1,1, \varepsilon)$ in rectangular coordinates. $\varepsilon$ is related to the shear angle $\alpha$ by

$$
\cos \alpha=(1+2 \varepsilon) /\left(2+\varepsilon^{2}\right)
$$

$\alpha$ is the angle between any two $a_{i}$. Values of $a_{i} a_{0}$ and $E$ are given in Table $I$.

The three reciprocal lattice vectors defined by

$$
\underline{g_{i}} \cdot \underline{a_{j}}=2 \pi \delta_{i j}
$$


Table I

Crystal structure Parameters of $\mathrm{Sb}$ at $4.2^{\circ} \mathrm{K}$

\begin{tabular}{|c|c|c|}
\hline $\mathbf{a}$ & $4.3007 \AA$ & Lattice constants in \\
\hline C & $11.222 \AA$ & hexagonal system \\
\hline $\mathrm{A}_{1}$ & $2.902 \AA$ & Nearest neighbor distance \\
\hline $\mathrm{A}_{2}$ & $3.343 \AA$ & $\begin{array}{l}\text { Next nearest neighbor } \\
\text { distance }\end{array}$ \\
\hline$\left|a_{i}\right|$ & $4.489 \AA=8.486$ a.u. & $\begin{array}{l}\text { Primitive lattice } \\
\text { parameter }\end{array}$ \\
\hline$a_{0}$ & 5.998 a.u. & \\
\hline$\alpha$ & $57^{\circ} 14^{\prime}$ & Rhombohedral angle \\
\hline$\varepsilon$ & 0.0416 & \\
\hline$\Omega_{0}$ & 404.7 a.u. & Volume of primitive cell \\
\hline$(2 \pi)^{3} / \Omega_{0}$ & $0.6130 \mathrm{a} . \mathrm{u}$ & $\begin{array}{l}\text { Volume of the Brillouin } \\
\text { zone }\end{array}$ \\
\hline $\mathbf{u}$ & 0.2336 & $\begin{array}{l}\text { Internal displacement } \\
\text { parameter }\end{array}$ \\
\hline$g_{0}$ & 0.5353 a.u. & \\
\hline$\left|g_{i}\right|$ & 0.9402 a.u. & $\begin{array}{l}\text { Length of primitive } \\
\text { reciprocal lattice } \\
\text { vectors }\end{array}$ \\
\hline$E_{F}$ & 0.4060 hartree & Free-electron Fermi energy \\
\hline $\mathbf{k}_{\mathrm{F}}$ & 0.9011 a.u. & $\begin{array}{l}\text { Free-electron Fermi } \\
\text { momentum }\end{array}$ \\
\hline
\end{tabular}




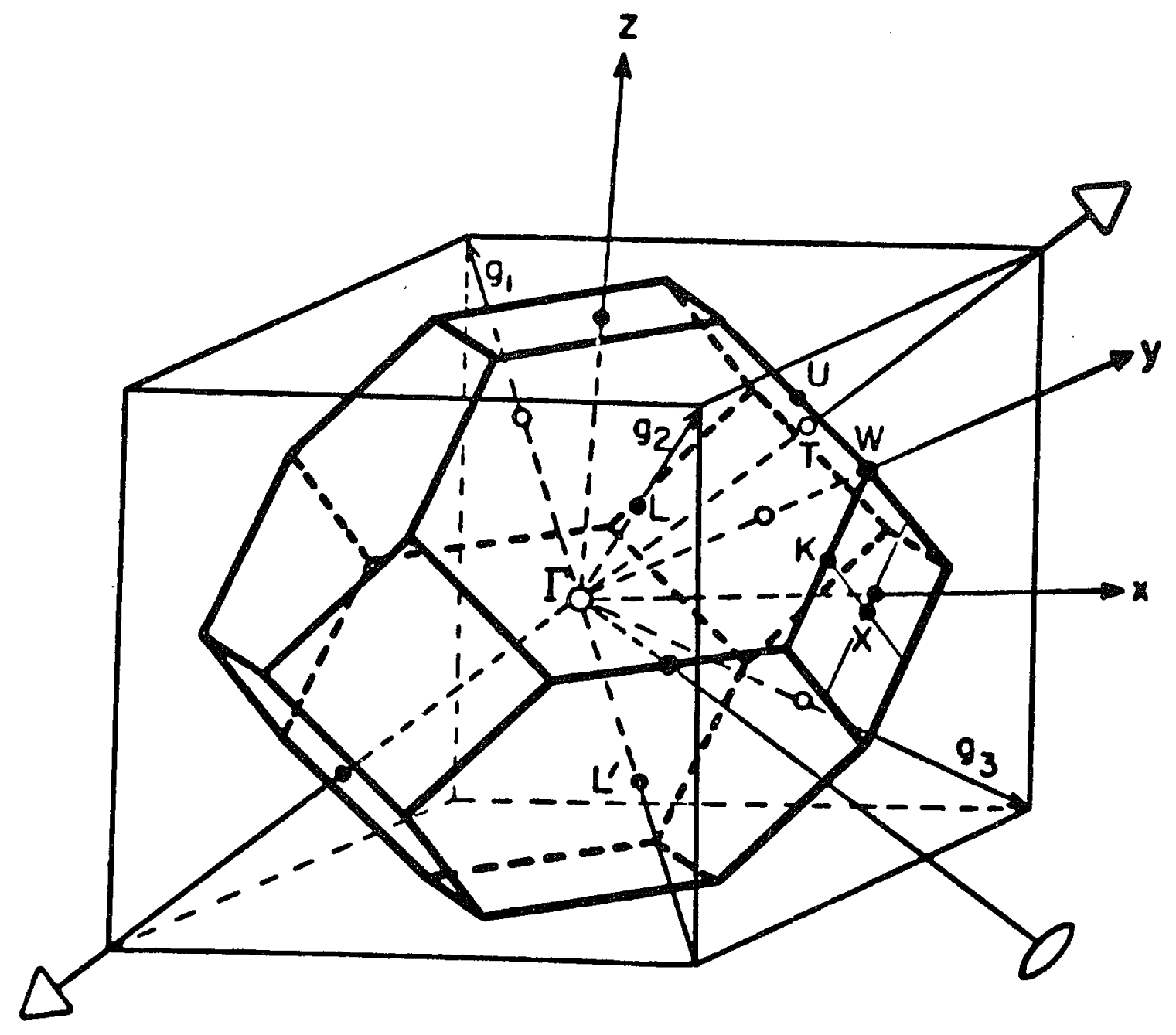

Fig. 2 
are given by

$$
\begin{aligned}
& \underline{g_{1}}=g_{0}(-(1+\varepsilon), 1,1), \\
& \underline{g_{2}}=g_{0}(1,-(1+\varepsilon), 1), \\
& \underline{g_{3}}=g_{0}(1,1,-(1+\varepsilon)),
\end{aligned}
$$

where $g_{0}$ is listed in Table $I$.

\section{Electron-Phonon Scattering}

In spite of the fact that the Fermi surface (FS) and band structure of antimony have been rather well mapped out, the elementary transport processes (e.g., the electrical and thermal conductivities and thermopower) are not fully understood. ${ }^{13}$ still open to interpretation are the scattering mechanisms in antimony, in particular, the electron-phonon (e-p) scattering.

In studying transport properties, the investigator is concerned with particles having velocities which are directed in a preferred direction under the action of external forces. The particles with which we are concerned here are electrons, holes, and phonons. The electrons and holes are described by Fermi surfaces and the phonons by experimentally determined dispersion relations. In the low-temperature range, the electrons and holes are scattered by other phonons, impurities, boundaries, and the electrons and holes. These scatterings (collisions) tend to bring the whole particle system back into equilibrium 
with the relaxation time being the essential parameter describing the process.

The parallel field radio frequency size effect (RFSE) has been used extensively for studying e-p collision frequencies in metals and semi-metals. 14 In a previous study into the temperature dependence of the electron and hole mean free paths in antimony, 15 the investigators concluded from the $\mathrm{T}^{2}$ dependence of the natural $\log$ of the amplitude of the RFSE signals that the e-p collision frequency is also proportional to $\mathrm{T}^{2}$.

The current investigation was undertaken to verify the $\mathrm{T}^{2}$ dependence of the signals but also to provide an opportunity to apply to antimony a parametrized scattering model which has been developed and described previously. 16 This model has worked very well in the case of copper, which displays the normal $\mathrm{T}^{3}$-dependent scattering behavior found in metals. It was assumed that if the e-p scattering rates in antimony were actually $\mathrm{T}^{3}$ plus some other effects which simply made the rates appeax proportional to $T^{2}$, then it should be possible to fit this model for antimony; and, in the process, some new information about the scattering process might be found.

The $\mathrm{T}^{3}$ dependence of the scattering frequency or mean-fxee path in a metal arises in the following manner. There are three principle factors which enter into determining the temperature-dependent mean-free path of an 
electron in a metal. 17 The first factor is the number of phonons available to cause the scattering. Since the electrons are confined to the Fermi surface and the temperature is low, this number is proportional to $\mathrm{T}^{2}$. The second factor is that the square of the matrix element for the interaction is proportional to the magnitude of the phonon wave vector $q$, and this leads to an additional $T$ dependence. These two factors give the $T^{3}$ law, and it is explicitly assumed that all scattering events are effective. If there is any angular dependence to the effectiveness, then the $\mathrm{T}^{3}$ law must be modified accordingly. For example, to obtain the resistivity, one must multiply by a factor of $(1-\cos \theta)$, where $\theta$ is the average angle through which the electron velocity direction is changed. At low temperatures, this leads to a $\mathrm{T}^{5}$ law for the resistivity of a metal.

While antimony exhibits the high temperature behavior of a metal, in the low temperature range the resistivity is more nearly proportional to $\mathrm{T}^{2}$ than to $\mathrm{T}^{5}$. If the dominant scattering process for electrons were electronelectron (e-e) rather than e-p scattering, a $\mathrm{T}^{2}$ dependence would be expected at low temperatures. However, all theories of the e-e scattering process predict that in antimony e-p scattering should dominate e-e scattering in the temperature range greater than $1 \mathrm{~K}$. 
In a previous attempt to explain this behavior of antimony, 18 the investigators based their defense of the quadratic $T$ results on an argument revolving around the nearly cylindrical nature of the FS of antimony. The current investigation chooses to take a different approach by fitting the RFSE data for antimony to the previously mentioned parametrized model and simply studying the results. 
CHAPTER II

\section{RADIO FREQUENCY SIZE EFFECT}

The data used in this investigation of the scattering rates in antimony was obtained by means of the radio frequency size effect (RFSE). The parallel field RFSE has been used extensively as a tool for studying e-p collision frequencies ${ }^{19}$ as well as for obtaining dimensions of Fermi surfaces of metals. ${ }^{20}$ The RFSE involves the detection of surface impedance changes when a magnetic field is applied parallel to the surface of a thin single crystal slab of a metal. In the presence of a magnetic field $\vec{H}$, the electrons in a metal move in reciprocal space on the FS in a plane perpendicular to the direction of $\vec{H}$. As the magnitude of the magnetic field is varied, the orbits of the electrons in the metal change in size. These extremal orbits will just fit inside of a sample consisting of a thick flat metal crystal of thickness $d$, when $\vec{H}$ is parallel to the sample surface, and when $k_{c}=(e / \hbar c) H d$, where $k_{c}$ is an extremal diameter of the FS, $e$ is the electron charge, $\hbar$ is Planck's constant divided by $2 \pi$, and $c$ is the speed of light. Such orbits are called parallel-field extremal orbits.

When an extremal diameter orbit exactly spans the sample thickness, a change in the radio-frequency surface impedance of the sample is observed. The strength of the 
magnetic field at that value can be directly related to the size of the FS. The amplitudes of these impedance changes depend on the probability of an electron traversing the sample thickness. This probability, in turn, depends on both the sample thickness and the sample temperature. By studying amplitude variations of the RFSE signals as a function of these two parameters, information can be obtained about the e-p and electron-impurity scattering rates, $\nu_{P}$ and $\nu_{I}$, in the metal. The specific design of the RFSE apparatus employed in this investigation will be discussed later in the experiment chapter.

An earlier RFSE study of antimony ${ }^{21}$ demonstrated that the amplitude of the RFSE signals exhibit a $\mathrm{T}^{2}$ dependence in the $1-4 \mathrm{~K}$ range. This fact led to the previously mentioned conclusion that the mean free path of carriers in antimony varies as $\mathrm{T}^{2}$. In the present investigation, the amplitudes of the RFSE signals were again found proportional to $\mathrm{T}^{2}$. However, due to a considerably different interpretation of the origin of this $\mathrm{T}^{2}$ behavior, the e-p scattering rate is found proportional to $T^{3}$, as is the case in other metals. The basis for this new interpretation lies in the realization that scattering effectiveness of different groups of phonons must be considered in analyzing the scattering process. This effectiveness will be discussed further in the next chapter. 
CHAPTER III

SCATTERING MODEL

The complete parameterized scattering model which is used in this analysis has been described previously ${ }^{22}$ and is listed in Appendix I. This paper will be referred to hereafter as KAG. The principle features of this model are summarized here, with exceptional points for antimony being noted.

The amplitude, A, of a parallel field RFSE signal is given by

$$
\begin{aligned}
A & =A_{0} \sum_{m=1}^{\infty} \exp \left(-m \pi \bar{v}_{\text {eff }} / \Omega\right) \\
& =A_{0}\left[\exp \left(\pi \bar{v}_{\text {eff }} / \Omega\right)-1\right]^{-1}
\end{aligned}
$$

where $\bar{\nu}_{\text {eff }}$ is the average effective collision frequency for all types of scattering along an orbit, $\Omega=\mathrm{eH} / \mathrm{m}^{*} \mathrm{c}$ is the cyclotron frequency, $\mathrm{m}^{*}$ the effective cyclotron mass, $\mathrm{H}$ the strength of the applied magnetic field, and $A_{0}$ is a constant. In the absence of e-e scattering the effective collision frequency is composed of $\nu_{I}$ and $\nu_{P}$, and Matthiessen's Rule is assumed.

The impurity scattering rate is assumed to be independent of temperature. It is included in the analysis by multiplying the calculated signal amplitude by

$$
\exp \left(-\pi \nu_{I} / \Omega\right)=z^{d}
$$


where $d$ is the sample thickness and $z$ is treated as an adjustable parameter in the data fitting process. The dependence on sample thickness arises from the fact that the cyclotron frequency at the field of the RFSE signal depends inversely on $d$. This expression differs by a factor of $\pi$ from KAG due to a misprint in that paper.

The matrix element, $\Gamma(\varepsilon, q)$, necessary to find the e-p interaction is given by

$$
\begin{aligned}
\Gamma(\varepsilon, q) & =\operatorname{cq}\left[\frac{2}{\exp \left(\beta h \omega_{q}\right)-1}+\frac{1}{\exp \left[\beta\left(\varepsilon+h \omega_{q}\right)\right]+1}\right. \\
& \left.+\frac{1}{\exp \left[\beta\left(-\varepsilon+h \omega_{q}\right)\right]+1}\right] \delta\left(h \omega_{q}-\varepsilon-\varepsilon^{\prime}\right),
\end{aligned}
$$

where $h w_{q}$ is the phonon energy, $q$ the magnitude of the phonon wave vector and $\varepsilon$ the electronic energy. The factor of $\mathrm{C}$ describes the strength of the interaction and is included as an adjustable parameter. In performing integrals over phonons, the approximation

$$
\int d^{3} \vec{q}=2 \pi \int_{0}^{2 q} F
$$

is used. Here $q$ is the wave vector of a spherical FS for the electron being scattered. This spherical Fs assumption is obviously not correct for the electron and hole pockets in antimony. However, several alternate procedures have been tried, such as an angular dependent $\mathrm{q}_{F}$, a cylindrical approximation for the integrals and a smooth cutoff for the upper limit and there has been found no significant 
improvement to the fit of the data. In addition, any additional complications in the form of these integrals lead to additional adjustable parameters which do not appear to be justified in fitting the present data. Not all phonons with $q<q_{F}$ are effective in scattering electrons. It has been pointed out by Gantmakher ${ }^{23}$ that electrons will not be removed from an RFSE signal by phonons with wave vector $q$ satisfying

$$
\frac{a}{q_{F}} \ll \frac{\delta}{d}
$$

where $\delta$ is the skin depth. Thus, in averaging the e-p matrix element over phonons, an effectiveness function, $\gamma(q)$, is included, where

$$
\gamma(q)=\tanh \left(\omega_{q} \alpha / \alpha\right)
$$

This is an approximation which is derived in KAG. Here $\alpha$ is an adjustable parameter used to fit the experimental data. It is shown in KAG that the effectiveness parameter, $\alpha$, is proportional to $\theta_{D} \delta$, where $\theta_{D}$ is the Debye temperature. Unlike metals like copper in antimony the skin depth is temperature dependent in the range 1-4 $\mathrm{K}$. We calculated the anomalous skin depth from the electrical conductivity data of $\mathrm{Tsai}^{24}$ at different temperatures in this range and fit the calculated points to the following function:

$$
\delta(T)=\left(1+1.8 / T^{3 \cdot 3}\right) \delta_{0}
$$

where $\delta_{0}$ is a constant. In the RFSE data fitting procedure 
a temperature dependent effectiveness parameter of the form

$$
\alpha(T)=\alpha * \delta(T) / \delta_{0}
$$

was used, with the adjustable parameter being $\alpha^{*}$.

Gantmakher ${ }^{25}$ has pointed out that phonons are

ineffective in scattering electrons when their wave vectors are larger than twice the k-space dimensions of the piece of FS under observation. This group of phonons is ineffective because momentum cannot be conserved in the scattering process. This is a particularly relevant point in studying antimony because the FS dimensions are small in comparison to phonon wave vector magnitudes in the temperature range of interest. The effect of this cutoff is to cause an effective Debye temperature, $\theta_{D}^{*}$, to exist which is much smaller than the temperature corresponding to the maximum phonon energy, $\theta_{D}$. The upper limit of the phonon averaging integral is the ratio of the maximum effective phonon wave vector, $q$, to the average $q$ at the temperature at which the integral is being evaluated. This upper limit is given by $\theta_{\mathrm{D}}^{*} / \mathrm{T}$.

When the effectiveness function is included in the average of $\Gamma(\varepsilon, q)$ over phonons,

$$
\langle\Gamma(\varepsilon)\rangle=\int_{0}^{q_{\max } / q} \Gamma(\varepsilon, q) \gamma(q) d^{3} q .
$$

With the substitution of variables $y=\beta \pi \omega_{q}$ and $x=\beta \varepsilon$, $\langle\Gamma(\varepsilon)\rangle$ is proportional to $\mathrm{T}^{3} \mathrm{H}(\mathrm{x})$, where 
$T^{3} H(x)=T^{3} \int_{0}^{\theta * / T} \tanh \left(\frac{T y d}{\alpha^{*}}\right) y^{2}\left(\frac{2}{e^{y}-1}+\frac{1}{e^{x+y}+1}+\frac{1}{e^{-x+y}+1}\right) d y$.

When $\theta_{D}^{*}$ is of order five times or less than the temperature range over which data are obtained, its precise value becomes an important factor in the data fitting procedure.

The final formula for a single pass of the electron across the sample must contain an average over electrons of different energies near the Fermi energy. This expression is obtained in KAG and is

$$
A_{1}=\frac{A_{0}}{2} \int_{0}^{\infty} \frac{z^{d} \exp \left[-d G T^{3} H(x)\right]}{\cosh ^{2}\left(\frac{x}{2}\right)} d x .
$$

This is the single pass formula for the RFSE amplitude, where $G$ is directly proportional to $v_{P}$ per $K^{3}$. A multiple pass expression is given in KAG and is obtained by a sum over $\mathrm{n}$ traverses of an electron across the sample. Note that the expression for $A_{1}$ contains four adjustable parameters: $G$, which is proportional to $\nu_{P}$ per $K^{3} ; z_{\text {, }}$ which depends on $\nu_{I} ; \alpha^{*}$, the effectiveness parameter; and $\theta_{D^{\prime}}^{*}$, the effective Debye temperature.

It is shown in KAG that a correction needs to be applied to the measured temperature dependent amplitudes due to the temperature dependence of the bulk conductivity in the skin depth. This correction is proportional to $\sigma \sqrt{\delta d}$, where $\sigma$ is the d.c. conductivity of the material. For metals $\delta$ is temperature independent and $\sigma \propto T^{5}$ in the temperature range of interest. In the present case, 
$\sigma \propto \mathrm{T}^{2}$ and $\delta$ is temperature dependent. We have added the following multiplication correction to the multiple pass formula:

$$
\exp \left\{Q T^{2}\left[d\left(1+1.8 / T^{3 \cdot 3}\right)\right]^{1 / 2}\right\}
$$

where $Q$ is treated as a fifth adjustable parameter. 
CHAPTER IV

EXPERIMENTAL PROCEDURE

All of the RFSE amplitude measurements were performed on a thin single crystal sample of antimony which was cut from a zone refined bar (quoted purity: 99.9999\%). The sample was oriented with the normal to its large surface in the bisectrix direction. The bar was initially mounted on a goniometer with silver conducting paint, which served both as a glue and as a means of electrical conduction between the sample and the goniometer. The electrical conduction was necessary for the spark erosion process which would follow the $x$-ray alignment process. (When larger pieces of antimony were used initially, we used Duco cement for the glue and simply painted a connecting band of silver paint for conduction from the sample to the goniometer.) The sample was then aligned appropriately via Laue back reflection $x$-ray techniques. A thin slab was cut from the sample by means of spark erosion using a horizontal wire. The slab was then mounted on the goniometer, realigned, and one side was spark planed to produce a very flat, smooth surface. The sample was removed from the goniometer and the face of the goniometer was planed so that the face of the goniometer would be as parallel as possible to the planing wheel. The sample was again mounted with the previously planed side against the 
goniometer surface, and the rough side of the sample was then planed. This latter process ensured that the two large surfaces of the slab would be as parallel as possible. Prior to each $x$-ray of the sample, after the spark erosion, the surface of the sample was lapped on a cloth in a solution containing equal parts of hydrofluoric, nitric, and acetic acids and water. This was done to remove any surface strain which was caused by the spark erosion.

At each stage in which an $x$-ray was performed, care was taken to insure that the orientation was within $\pm 0.5^{\circ}$ of the desired direction. The finished sample was also checked to this tolerance.

Attention should be drawn to the fact that all of this care did not guarantee that a sample would be produced which would yield RFSE signals. Many samples were cut at various orientations which appeared to be good single-crystal samples with good surfaces under $x$-ray investigation, which nevertheless failed to yield RFSE signals. These "signal-less" samples were always $\mathrm{x}$-rayed again upon removal from the RFSE apparatus and were always found to again yield good $x$-ray photos. Antimony is easily cleaved perpendicular to the trigonal axis. Any slight mishandling during the orienting and cutting process could have caused internal slippage parallel to the cleavage planes which would not be detectable as an $x$-ray 
spot distortion. However the electron scattering from these planes of dislocation would completely destroy any RFSE signal.

RFSE measurements were performed at three different sample thicknesses which were obtained by repeated thinning of the original sample. The three thicknesses employed were $0.592 \mathrm{~mm}, 0.347 \mathrm{~mm}$, and $0.238 \mathrm{~mm}$. After measurements were performed on the original slab (0.592 $\mathrm{mm})$, the slab was spark planed to $0.347 \mathrm{~mm}$ by the previously outlined method, complete with the lapping and $\mathrm{x}$-ray procedures. This was repeated after the second set of RFSE measurements in order to obtain the $0.238 \mathrm{~mm}$ thickness set of measurements.

It should be noted that samples this thin were mandated by the necessity to have the small caliper values in antimony occur at values of the applied magnetic field which were large compared to the inhomogeneities in magnetic field existing over the sample volume.

The experimental apparatus which was used is similar to that described by steinhaut and Goodrich ${ }^{26}$ using the radio oscillator and detection circuit of Ruthruff and Goodrich. 27 This apparatus allows both the first and second derivatives of the real part of the surface impedance to be recorded. The experimental apparatus is represented schematically in Fig. 3. The tank coil of the r.f. oscillator which consists of approximately 50 turns 


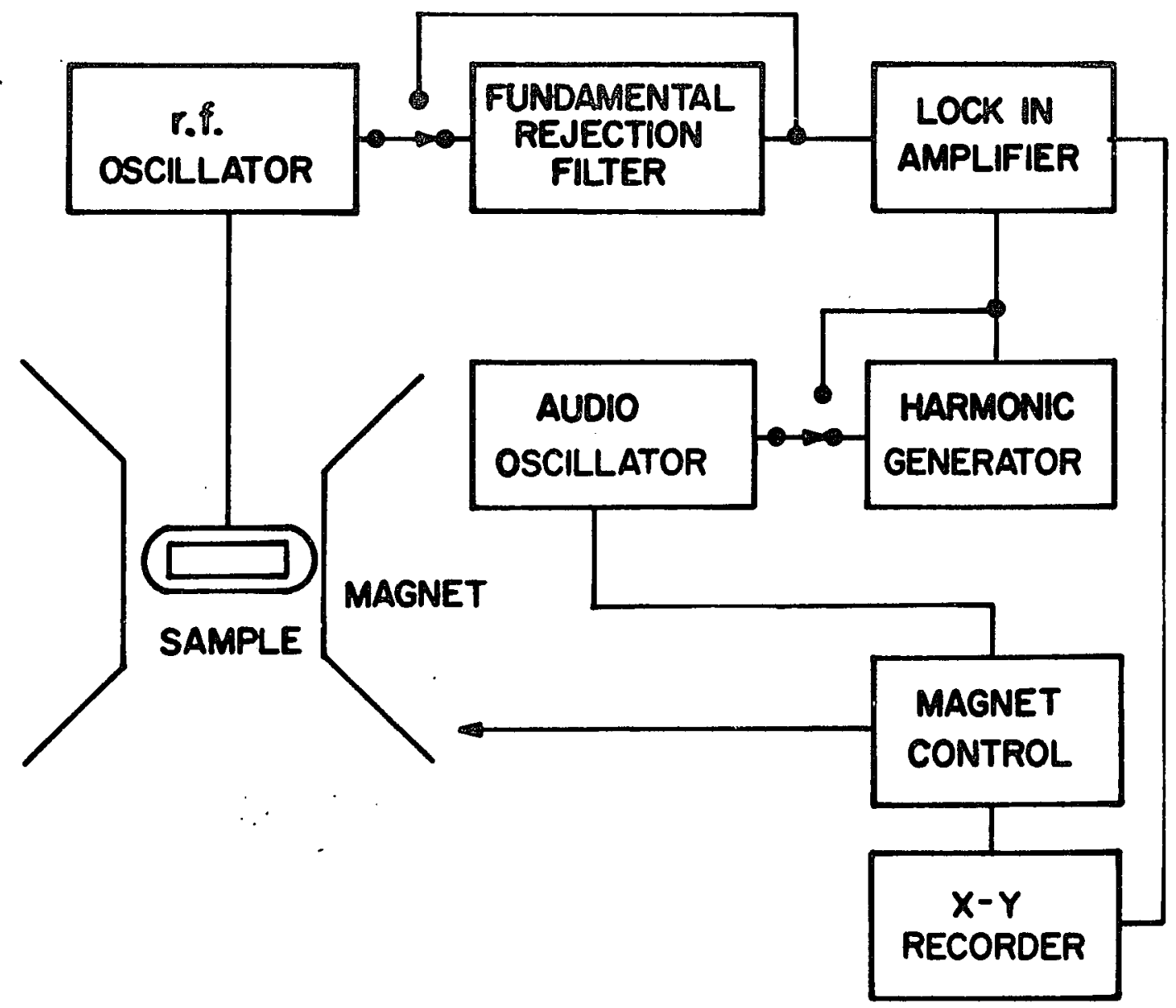

Fig. 3 
of number 40 copper wire. This coil was wound on an aluminum form having dimensions slightly larger than the antimony sample dimensions. The form was then removed and the sample inserted in its place. The sample-coil configuration then acted as the inductive element of the oscillator. Therefore, when the characteristics of the sample are affected by an applied magnetic field, the inductance of the sample-coil combination changes; and the changes are noted by the detection circuit.

A different tank coil was used for each sample thickness in order to establish a good filling factor for the sample in the coil. This proved to be beneficial in improving the signal to noise ratio of the signals. The r.f. frequency used was approximately $9 \mathrm{MHz}$.

The sample coil and dewar system were located in the gap of a Helmholtz coil pair which provided a horizontal magnetic field in the plane of the sample. The sample normal, $n$, is vertical in this arrangement; and the magnitude of the magnetic field can be varied with time as is required for RFSE measurements. The main field was varied from 0 to $200 \mathrm{G}$.

When working at low values of magnetic field such as is necessary with antimony, small unwanted fields such as those generated by the earth and by electrical equipment cannot be ignored. Therefore, in order to have as near as possible a magnetic field equal to zero in the vicinity of 
the sample prior to the application of the principle magnetic field, three mutually perpendicular pairs of coils were placed around the Helmholtz coils to generate magnetic fields which would compensate for any external magnetic fields. With a gaussmeter placed at the location of the sample, the power supplies for these three coil pairs were adjusted until a field of less than 0.01 G was obtained at the sample position. The three coil sets were checked and adjusted periodically to be certain that they were still performing their intended function.

The sample was sealed inside a double-walled can arrangement, which is diagrammed in Fig. 4. The helium exchange gas between the sample and the inner can allows for a limited amount of heat transfer between the sample and the liquid helium bath. The bath temperature was adjusted between 1.2 and $4.2 \mathrm{~K}$ by pumping on the bath. Due to the heat leak between the sample and the room environment, the sample temperature actually rose above $4.2 \mathrm{~K}$ at the end of data runs; so some of the data was taken at temperatures above the bath temperature. All amplitude data was taken between 1.2 and $4.4 \mathrm{~K}$. 


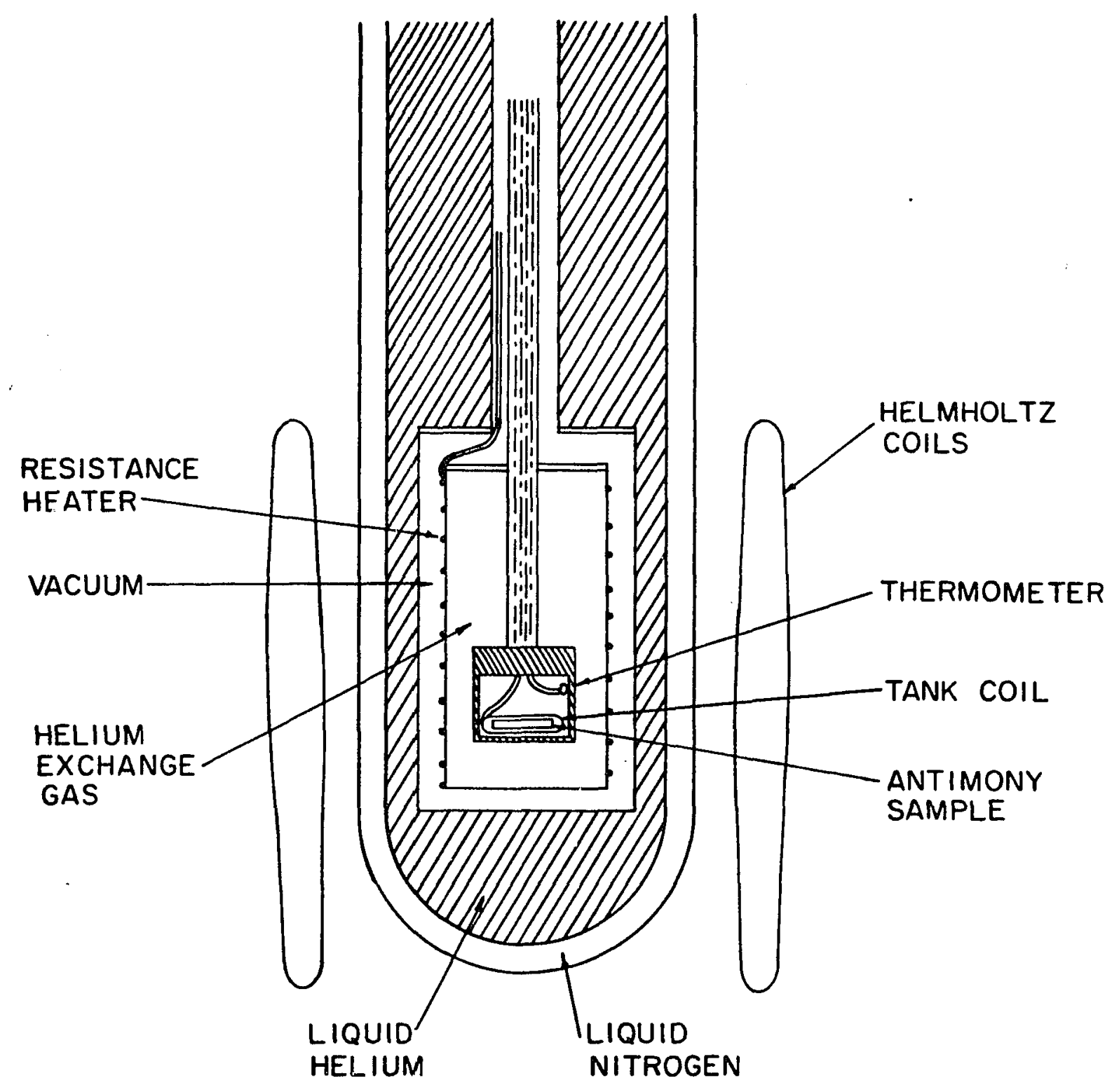

Fig. 4 
CHAPTER V

EXPERIMENTAL RESULTS

A typical set of raw data is shown in Fig. 5. The RFSE amplitudes were determined from the peak to peak amplitudes of the $\mathrm{dR} / \mathrm{dH}$ vs. $\mathrm{H}$ signals at each temperature. Data similar to this were obtained for several orbits on both the electron and hole surfaces in the bisectrix plane. The FS of antimony was discussed to some extent in the introduction to this dissertation and is discussed more completely in Herrod, Gage, and Goodrich ${ }^{28}$ or in Herrod. 29 The FS is reproduced in Fig. 6. Outlines of two of these pieces of FS projected onto the bisectrix plane are shown in Fig. 7 along with an indication of the orbits on which data were obtained in this investigation. Data also were obtained on a second sheet of electron surface which is rotated from these projections and appears nearly end on in this plane. These data also were indicated by closed circles in Fig. 7.

The gaps in the data for some angles are due to the overlapping of two or more signals. Not only is there a great deal of overlap among the basic signals in antimony, but many of the double-field signals overlap the basic signals and are of significant size to add to the difficulty of separating the various signals. When this occurs, the amplitudes cannot be determined accurately. For each 


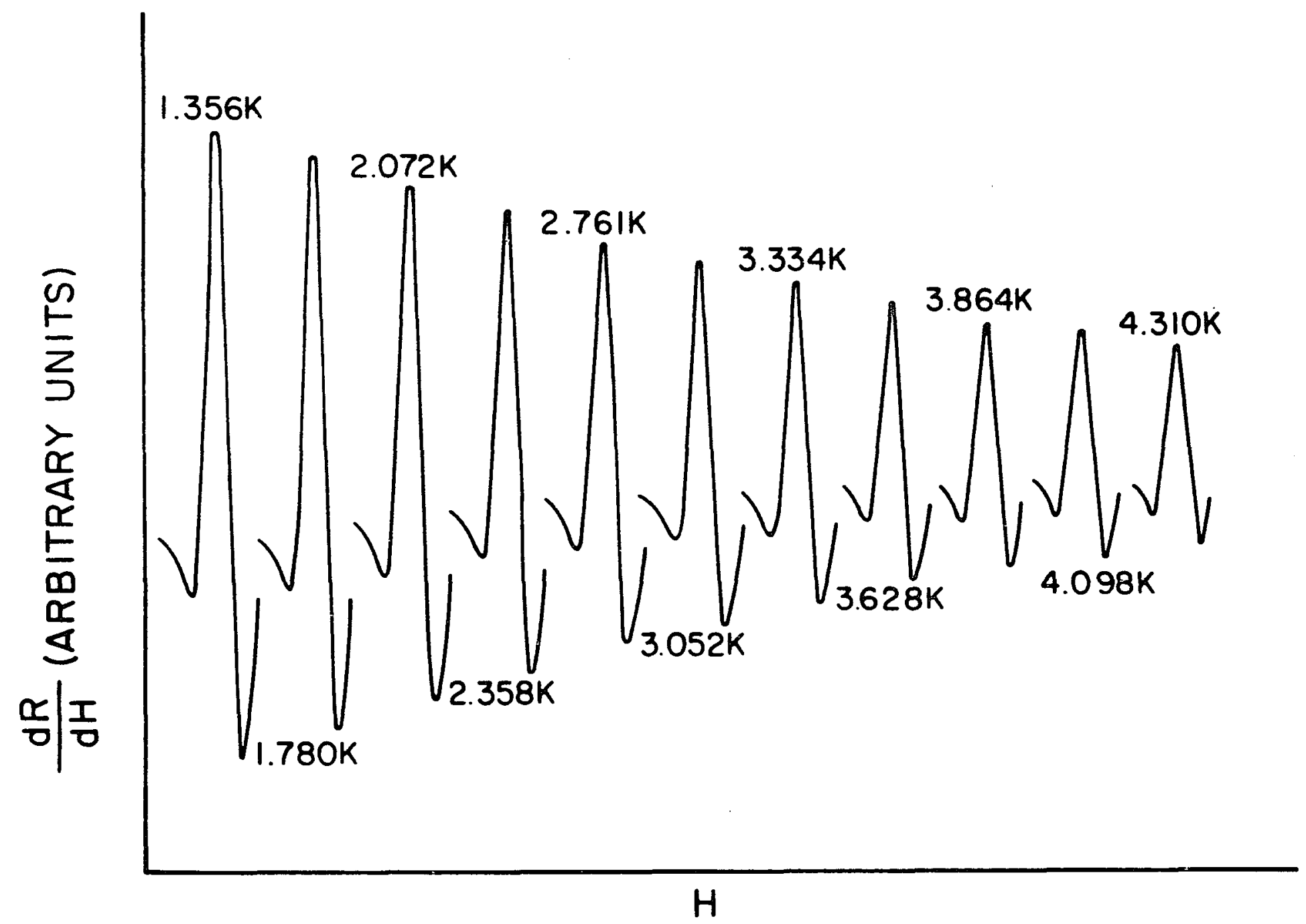

Fig. 5 


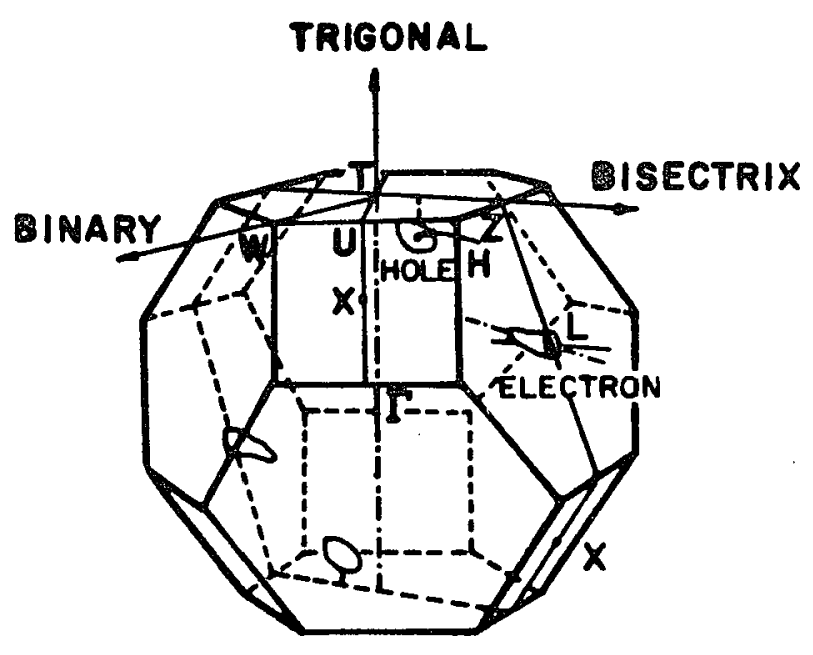

Fig. 6 


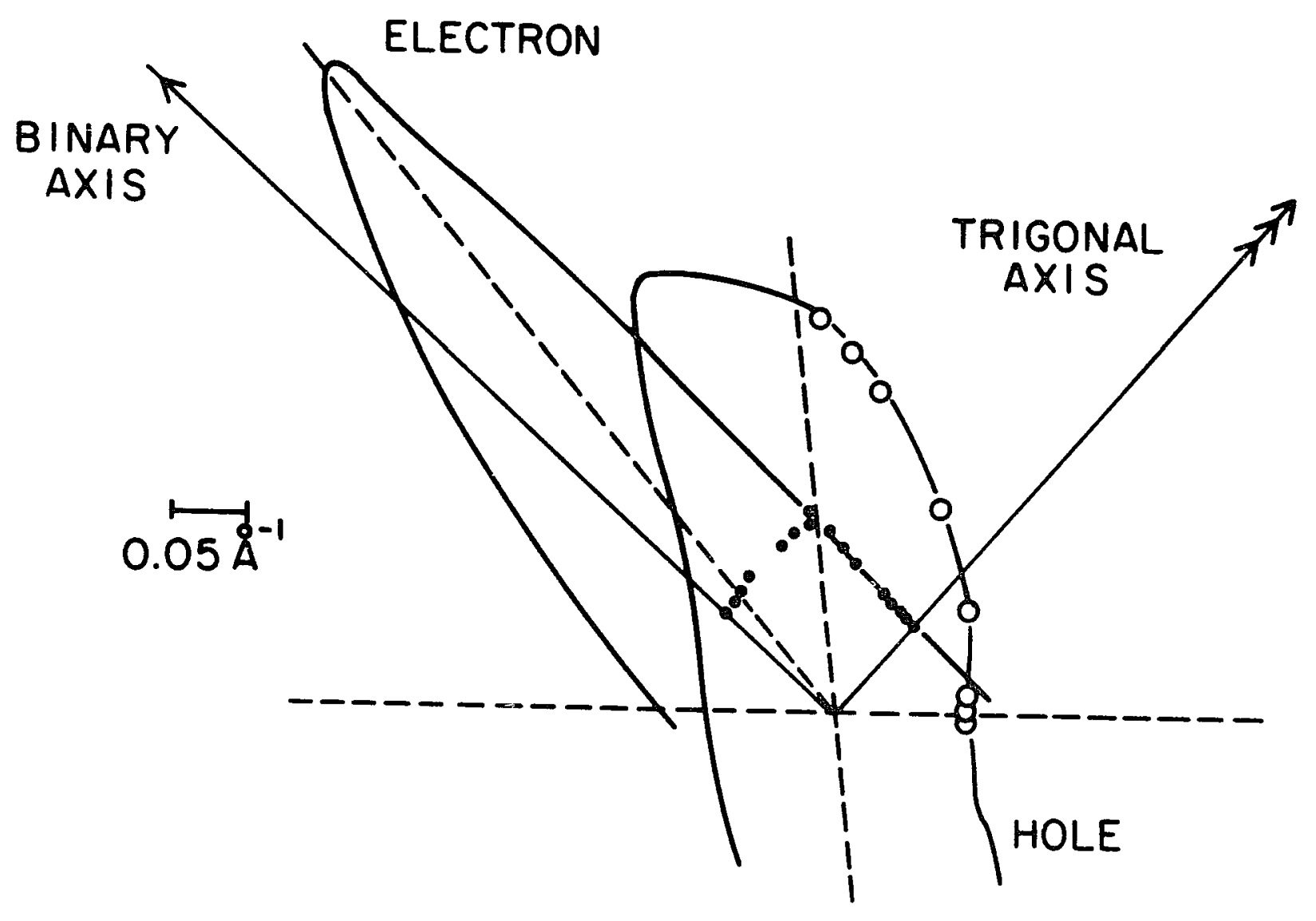

Fig. 7 
of the measurement angles and for each set of signals the natural log of the amplitude at each temperature was taken and plotted against the square of the temperature. The most probable slopes of these lines were found by the method of least squares, and these slopes were used as input data for the analysis. A listing of the slopes along with the effective masses for the orbits is given in Table II for each measurement angle. All of the data in Table II was taken on the thinnest sample, $d=0.238 \mathrm{~mm}$. For the magnet angle $\theta=10^{\circ}$ from the trigonal, data were obtained for three sample thicknesses, $d=0.592 \mathrm{~mm}$, $0.347 \mathrm{~mm}$, and $0.238 \mathrm{~mm}$. The three-thickness data were obtained for both a hole and an electron and are listed in Table III.

One particularly interesting multiple signal effect was observed. Occasionally the amplitude of an apparently normal signal actually increased in size as the temperature was increased in the process of data acquisition. A sample of these types of data is reproduced in Fig. 8. This, of course, is directly opposite to what is expected and normally occurs. Although there is no detailed explanation for this phenomena, it appears to be related to the overlap of multiple signals, as this only occurred where two or more signals did overlap.

Normally, if it was apparent that two or more signals were mixed, the amplitude vs. temperature data were not 


\section{Table II}

Measured slopes of $\ln \left(A / A_{0}\right)$ vs. $T^{2}$ and effective masses* for orbits in antimony. All magnet angles, $\theta$, are measured from the trigonal toward the binary.

\begin{tabular}{|c|c|c|c|c|c|c|c|c|}
\hline & \multicolumn{2}{|c|}{ Electrons } & \multicolumn{3}{|c|}{ Holes } & \multicolumn{3}{|c|}{$\begin{array}{l}\text { Electrons } \\
\text { (end on) }\end{array}$} \\
\hline$\theta$ & slope & $m^{*} / m_{0}$ & $\theta$ & slope & $\mathrm{m}^{*} / \mathrm{m}_{\mathrm{o}}$ & $\theta$ & Slope & $\mathrm{m}^{*} / \mathrm{m}_{\mathrm{o}}$ \\
\hline 0 & 0.060 & 0.100 & -50 & 0.048 & 0.070 & 50 & 00.23 & 0.380 \\
\hline 5 & 0.060 & 0.100 & -45 & 0.047 & 0.065 & 55 & 0.23 & 0.370 \\
\hline 10 & 0.064 & 0.105 & -40 & 0.054 & 0.065 & 60 & 0.22 & 0.360 \\
\hline 15 & 0.064 & 0.110 & -10 & 0.052 & 0.070 & 75 & 0.23 & 0.320 \\
\hline \multirow[t]{2}{*}{20} & 0.064 & 0.110 & 15 & 0.083 & 0.100 & 80 & 0.17 & 0.320 \\
\hline & & & & & & 85 & 0.14 & 0.320 \\
\hline 35 & 0.070 & 0.130 & 35 & 0.085 & 0.170 & 90 & 0.12 & 0.320 \\
\hline 40 & 0.072 & 0.135 & 40 & 0.089 & 0.195 & & & \\
\hline 45 & 0.083 & 0.150 & 45 & 0.118 & 0.220 & & & \\
\hline 50 & 0.082 & 0.160 & & & & & & \\
\hline
\end{tabular}

*Datars and Vanderkooy (1964). 


\section{Table III}

Measured slopes of $\ln \left(A / A_{0}\right)$ vs. $T^{2}$ for orbits in antimony. All measurements made in the plane normal to the bisectrix axis at $\theta=10^{\circ}$ from the trigonal.

\begin{tabular}{ccc}
$\begin{array}{c}\text { Thickness } \\
(\mathrm{mm})\end{array}$ & Electron & Hole \\
\hline 0.592 & 0.200 & 0.116 \\
0.347 & 0.120 & 0.082 \\
0.238 & 0.064 & 0.052 \\
\hline
\end{tabular}




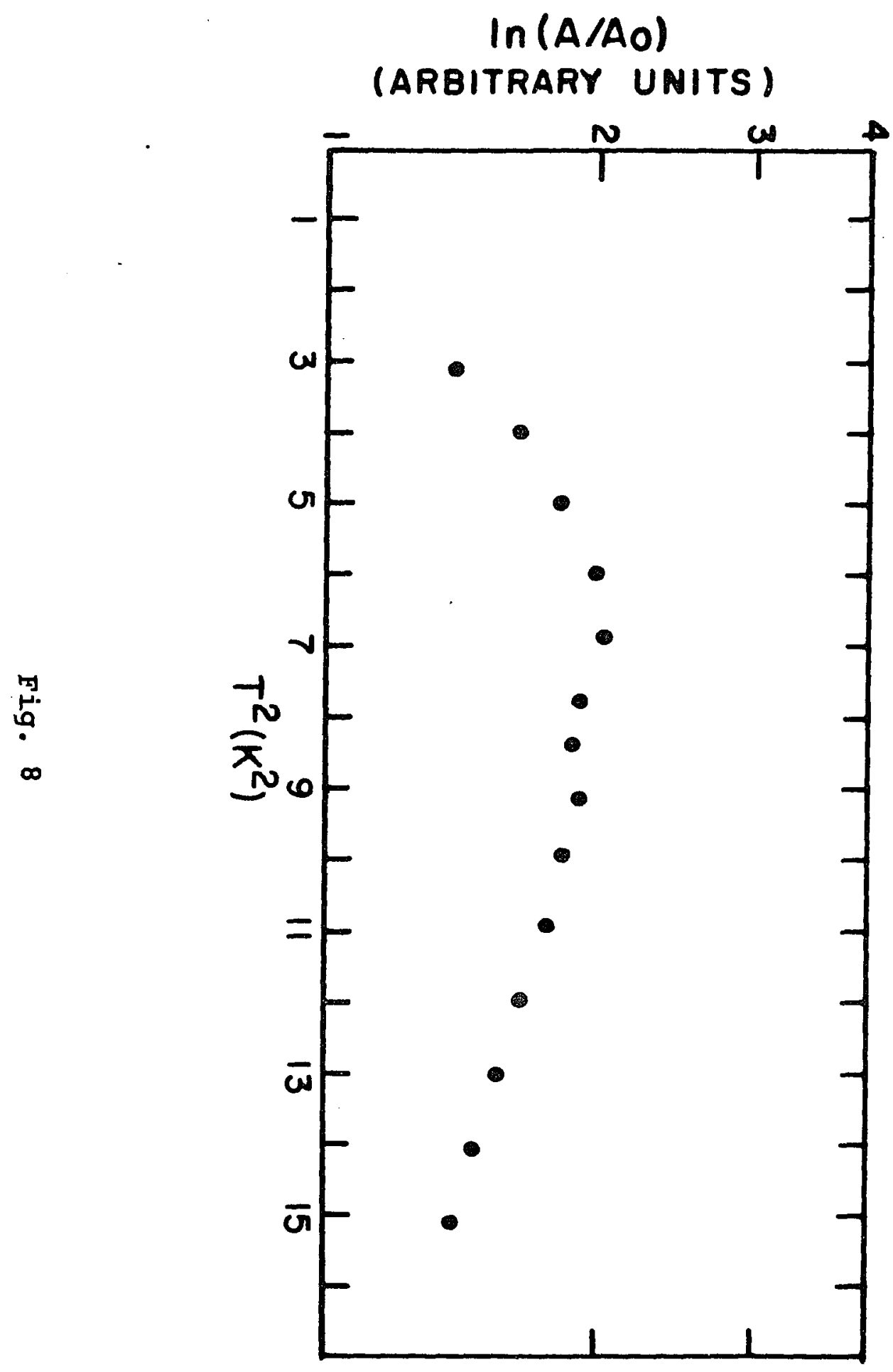


even measured. But occasionally a very clear signal which appeared to contain only one signal was analyzed; and the $\ln \left(\mathrm{A} / \mathrm{A}_{0}\right)$ vs. $\mathrm{T}^{2}$ produced this anomalous behavior. Each time this behavior was noted, however, it could be shown that the data point actually occurred where two or more signals overlapped. It is possible that differing signals with differing amplitudes and differing slopes of their In $\left(A / A_{0}\right)$ vs. $T^{2}$ lines could combine to produce this behavior. However, the effect was seldom noted and little data were recorded at these types of points.

Thus, the initial step in the analysis of the signals was to actually separate the different signals which appeared in the recorder traces. As mentioned, the number of usable signals is reduced considerably from the number of recorded signals since, in order to determine the amplitude of the signal, a certain amount of uninterrupted width of the signal must be seen. The signals in antimony are quite cluttered with many different basic field and double field signals overlapping. Each time that the overlapping signals occurred, all signals involved in the overlap had to be discarded because there was no reasonable way to resolve the contribution to the net signal from each of the individual signals. Because of this complicating factor many of the experimental signals were discarded. 
CHAPTER VI

ANALYSIS OF EXPERIMENTAL DATA

Once the data points wich are listed in Table III were acquired, the initial computational step was to fit the data from the three different sample thicknesses at one magnet angle in order to determine the five adjustable parameters. Once again, these are: $G$, which is proportional to $v_{P}$ per $\mathrm{K}^{3} ; \mathrm{z}$, which depends on $v_{I^{\prime}} \alpha^{*}$, the effectiveness parameter; $\theta_{D}^{*}$ the effective Debye temperature; and $Q$, which is a constant in the multiple pass correction. There are forty-five data points - fifteen temperatures at three thicknesses used as input for this process. In this parameter fitting procedure the different parameters have different effects on the calculated slopes and $\ln \left(A / A_{0}\right)$ vs. $\mathrm{T}^{2}$ shapes. The $\mathrm{z}$ parameter, impurity scattering, has the most effect on the spacing between the slopes of the different thickness samples. The effective Debye temperature, $\theta_{D^{*}}^{*}$ is adjusted until the calculated values of In $\left(A / A_{0}\right)$ obey a $T^{2}$ dependence over most of the temperature range. It must be remembered that the experimental values of $\ln \left(A / A_{0}\right)$ obey a $T^{2}$ dependence, so that the theoretical fit must fit the experimental fit. The effectiveness parameter, $\alpha^{*}$, has its greatest effect on the calculated data at the low temperature end of the range, although it does cause the spacing between sample thicknesses to 
change slightly. The main effect of $Q$ is to change the high temperature calculated points. The G parameter, ${ }_{P}$ ' shifts all of the calculated slopes by a similar amount. The FORTRAN computer program for this section of the analysis is Iisted in Appendix II.

The starting values for the parameters were estimated as follows: $\theta_{D}^{*}$ was set within the limits for both electrons and holes given by Tsai, ${ }^{30} \mathrm{G}$ was estimated from the data of Gantmakher and Dolgopolov, ${ }^{31} \alpha$ was estimated from the product $\theta_{D} \delta, z$ was set such that all sample thicknesses were in the single pass limit, and $Q$ was set equal to zero. Each of the parameters was then varied until a minimum deviation from the experimental slopes for all of the data was achieved.

Initially, the data was fit with a temperature independent skin depth; and when the best fit was obtained, the average deviation of the calculated slopes from the experimental slopes was approximately 15\%. The introduction of the temperature dependent skin depth immediately reduced this deviation to below 10\%. In the final parameter adjustment, the overall fit for the experimental data is $4.5 \%$ for the electrons and $3.4 \%$ for the holes. These values are within the uncertainty in the determination of the experimental slopes which was about $5 \%$. 
The following set of values for the parameters was obtained in this manner for $\theta=10^{\circ}$ from the trigonal axis. Along with each parameter is listed the average deviation of the calculated slopes from the experimental slopes which occur if this parameter were varied by $5 \%$ from the best fit value.

$$
\begin{aligned}
& \text { Holes: } \quad G=0.0049 \quad 4.8 \% \\
& \begin{array}{ll}
\mathrm{z}=0.0340 \quad 3.5 \text { 옹 }
\end{array} \\
& \theta_{\mathrm{D}}^{*}=12.0 \mathrm{~K} \quad 6.6 \% \\
& \alpha^{*}=5.0 \quad 4.2 \text { 응 } \\
& Q=0.075 \quad 5.7 \% \\
& \text { Electrons: } \quad \mathrm{G}=0.0121 \quad 7.2 \% \\
& \mathrm{z}<3.4 \times 10^{-6} \\
& \theta_{\mathrm{D}}^{*}=13.0 \mathrm{~K} \quad 8.1 \% \\
& \alpha^{*}=4.8 \quad 6.8 \% \\
& Q=0.075 \quad 6.9 \%
\end{aligned}
$$

All of the electron orbits for all of the sample thicknesses are in the single pass limit so only a lower limit on the impurity scattering rate can be established in this case. Approximately seven passes contribute appreciably to the signal for the thinnest sample in the case of the holes.

Once this parameter adjustment was completed, four of the five parameters in each case were held constant; and the value of $G\left(\propto \bar{v}_{P} / T^{3}\right)$ was adjusted to fit the measured slopes at all of the other magnet angles. The e-p and 
impurity scattering rates are computed as follows.

$$
\bar{v}_{P} / T^{3}=G \times 8.414\left(k_{c} h / m * \pi\right)
$$

and

$$
\bar{v}_{I}=-\left(k_{c} h / m^{*} \pi\right) \ln Z
$$

The values of the scattering frequencies that are obtained in this manner are the values at the Fermi energy. The scattering frequencies for each angle are listed in Tables IV-VI. It should be noted that while $\mathrm{Z}$ was not changed as a function of angle for the holes, $\bar{\nu}_{I}$ changes because $\mathrm{m}^{*} / \mathrm{m}_{\mathrm{o}}$ and $\mathrm{k}_{\mathrm{c}}$ are functions of angle. 


\section{Table IV}

Electron-phonon and impurity scattering rates for orbits in the bisectrix plane on the hole surface of antimony. Magnet angles, $\theta$, are measured from the trigonal axis. Effective masses are from Datars and Vanderkooy (1964).

\begin{tabular}{ccccc}
\hline$\theta$ & $k_{C}\left(\AA^{-1}\right)$ & $\mathrm{m} * / \mathrm{m}_{0}$ & $\bar{\nu}_{\mathrm{P}} / \mathrm{T}^{3}\left(10^{7} \mathrm{~s}^{-1} \mathrm{~K}^{-3}\right)$ & $\bar{\nu}_{\mathrm{I}}\left(10^{9} \mathrm{~s}^{-1}\right)$ \\
\hline-50 & 0.081 & 0.070 & 1.29 & 1.44 \\
-45 & 0.080 & 0.065 & 1.18 & 1.53 \\
-40 & 0.080 & 0.065 & 2.02 & 1.53 \\
-10 & 0.104 & 0.070 & 2.26 & 1.85 \\
15 & 0.145 & 0.100 & 7.15 & 1.81 \\
35 & 0.206 & 0.170 & 6.20 & 1.51 \\
40 & 0.229 & 0.195 & 6.52 & 1.46 \\
45 & 0.253 & 0.220 & 10.9 & 1.43 \\
\hline
\end{tabular}


Table V

Electron-phonon scattering rates for electron orbits in the bisectrix plane of antimony. Magnet angles are measured from the trigonal axis. Effective masses are from Datars and Vanderkooy (1.964).

\begin{tabular}{cccc}
\hline$\theta$ & $k\left(\AA^{-1}\right)$ & $\mathrm{m}^{*} / \mathrm{m}_{\mathrm{o}}$ & $\bar{\nu}_{\mathrm{P}} / \mathrm{T}^{3}\left(10^{7} \mathrm{~s}^{-1} \mathrm{~K}^{-3}\right)$ \\
\hline 0 & 0.075 & 0.100 & 2.44 \\
5 & 0.075 & 0.100 & 2.37 \\
10 & 0.075 & 0.105 & 2.68 \\
15 & 0.076 & 0.110 & 2.55 \\
30 & 0.078 & 0.110 & 2.64 \\
40 & 0.093 & 0.130 & 3.17 \\
45 & 0.101 & 0.135 & 3.50 \\
50 & 0.114 & 0.150 & 4.64 \\
\hline
\end{tabular}


Table VI

Electron-phonon scattering rates for the end on electron surface in the bisectrix plane. Magnet angles, $\theta$, are measured from the trigonal axis. Effective masses are from Datars and Vanderkooy (1964).

\begin{tabular}{cccc}
\hline$\theta$ & $k_{C}\left(\AA^{-1}\right)$ & $m^{*} / m_{0}$ & $\bar{\nu}_{\mathrm{P}} / \mathrm{T}^{3}\left(10^{7} \mathrm{~s}^{-1} \mathrm{~K}^{-3}\right.$ \\
\hline 50 & 0.121 & 0.380 & 3.48 \\
55 & 0.116 & 0.370 & 4.45 \\
75 & 0.112 & 0.360 & 5.59 \\
80 & 0.103 & 0.325 & 8.15 \\
85 & 0.098 & 0.320 & 7.68 \\
90 & 0.097 & 0.320 & 7.73 \\
\hline
\end{tabular}




\section{CHAPTER VII}

\section{CONCLUSIONS}

Inspection of Tables IV-VI shows that the electronphonon scattering rates in antimony are highly anisotropic. The values given in these tables are evaluated at the Fermi energy. They have been determined from the energy averaged values which enter into the experimental RFSE slopes. Based on the fact that adjustment of the effective Debye temperature, $\theta_{D^{*}}^{*}$ allowed fitting of the calculated values of $\ln \left(\mathrm{A} / \mathrm{A}_{0}\right)$ to a $\mathrm{T}^{2}$ dependence, it is our interpretation that the $\mathrm{T}^{2}$ dependence which is experimentally observed arises due to the low $\theta_{D}^{*}$ rather than because of a cylindrical FS or electron-electron scattering. The final calculated scattering frequencies are proportional to $\mathrm{T}^{3}$ as is the case for e-p scattering in most metals.

Different impurity scattering rates are obtained for electrons and holes. The sample thickness was sufficiently great that all electron orbits fell into a single pass limit. The lighter, smaller holes made multiple passes on the average and thus had a smaller value of $\bar{v}_{I}$ than the heavier, larger electrons.

The values of the effective Debye temperatures which best fit this data are well within the range given by 
Tsai, 32 which is necessary if the transport properties of antimony in this temperature range are to be explained. Finally, since the low effective Debye temperatures correspond to a low effective phonon wave vector, the present results are a direct confirmation of the fact that phonons of wave vector greater than $2 \mathrm{k}_{\mathrm{F}}$ are ineffective in scattering electrons. 


\section{REFERENCES}

1. Y. Eckstein, Phys. Rev. 129, 12 (1963).

2. I. Eriksson, O. Beckman, and S. Hornfeldt, J. Phys. Chem. Solids 25, 1339 (1964).

3. J. B. Ketterson, Phys. Rev. 129, 18 (1963).

4. A. P. Korolyuk and L. Ya. Matsakov, Sov. Phys. - JETP 25, 270 (1967) .

5. G. N. Rao, N. H. Zebouni, C. G. Grenier, and J. M. Reynolds, Phys. Rev. 133, Al41 (1964).

6. I. S. Lerner and P. C. Eastman, Can. J. Phys. 41, 1523 (1963)

7. W. R. Datars and J. Vanderkooy, IBM J. Res. Develop. B. 247 (1964).

8. L. R. Windmiller, Phys. Rev. 149, 472 (1966) and L. R. Windmiller and M. G. Priestley, solid state Comm. $\underline{3}$, 199 (1965).

9. I. M. Falicov and P. J. Lin, Phys. Rev. 141, 562 (1966).

10. R. A. Herrod, C. A. Gage, and R. G. Goodrich, Phys. Rev. B 4, 1033 (1971).

11. H. Suematsu, N. Koshino, and S. Tanuma, J. Low Temp. Phys. 15, 281 (1974).

12. R. A. Herrod, PhD. Dissertation, Louisiana State University (1971).

13. J-P. Issi, Aust. J. Phys. 32, 585 (1979).

14. V. F. Gantmakher, Rep. Prog. Phys. 37, 317 (1974). 
15. V. F. Gantmakher and V. T. Dolgopolov, Sov. Phys. JETP 33, 1215 (1971).

16. J. C. Kimball, L. W. Adams, and R. G. Goodrich, Phys. Rev. B 19, 2905 (1979).

17. J. M. Ziman, Electrons and Phonions (Oxford U. P., Oxford, 1963).

18. V. F. Gantmakher and V. T. Dolgopolov, Sov. Phys. JETP 33, 1215 (1971).

19. V. F. Gantmkaher, Rep. Prog. Phys. 37, 317 (1974)

20. P. B. Visscher and L. M. Falicov, Phys. Status Solidi 54, 9 (1972).

21. V. F. Gantmakher and V. T. Dolgopolov, Sov. Phys. JETP $\underline{33}, 1215$ (1971).

22. J. C. Kimball, L. W. Adams, and R. G. Goodrich, Phys. Rev. B 19, 2905 (1979).

23. V. F. Gantmakher, Rep. Prog. Phys. 37, 317 (1974).

24. G. I. Tsai, PhD. Dissertation, Louisiana State University (1974).

25. V. F. Gantmakher, Rep. Prog. Phys. 37, 317 (1974).

26. O. I. Steenhaut and R. G. Goodrich, Phys. Rev. B I, 4511 (1970).

27. T. I. Ruthruff and R. G. Goodrich, J. of Phys. E, Vol. 12, No. 2, p. 94 (1979).

28. R. A. Herrod, C. A. Gage, and R. G. Goodrich, Phys. Rev. B $\underline{4}, 1033$ (1971).

29. R. A. Herrod, PhD. Dissertation, Louisiana State University (1971). 
30. G. I. Tsai, PhD. Dissertation, Louisiana State University (1974).

31. V. F. Gantmakher and V. T. Dolgopolov, Sov. Phys. JETP 33, 1215 (1971).

32. G. I. Tsai, PhD. Dissertation, Louisiana State University (1974). 
APPENDIX I

\section{A. Scattering Model}

The purpose of the exercise which follows is to develop a more complete parametrized model with which parallel field RFSE temperature-dependent data can be analyzed. Therefore, we give the elements of the model and how they are folded into a data fitting procedure. The physical quantities obtained from this process and their implications are also discussed. Sections 1-8 below describe the elements of our model.

1. The impurity scattering rate is assumed to be independent of temperature. If $\nu_{I}$ is the impurity scattering rate, then the RFSE signal amplitude is multiplied by $\exp \left(-v_{I} / \Omega\right)$ and we set

$$
\exp \left(-v_{I} / \Omega\right)=z^{d}
$$

where $\mathrm{Z}$ is treated as an adjustable parameter in the data fitting process from which $\nu_{I}$ can be obtained. Note that $\Omega \propto H \propto 1 / d$ for the RFSE signal.

2. The electron-phonon interaction involving the electrons contributing to the signal includes the fact that the scattering rate is dependent on an individual electron's energy, $\varepsilon$, relative to the Fermi energy, $E_{F}$. As was previously shown by Gantmakher and Wagner and Albers, the e-p scattering rate due to the processes shown in 
Fig. 9 is given by

$$
\begin{aligned}
\Gamma(\varepsilon, q) & =\operatorname{cq}\left[\frac{2}{\exp \left(\beta \hbar \omega_{q}\right)-1}+\frac{1}{\exp \left(\beta\left(\varepsilon+\hbar \omega_{q}\right)\right)+1}\right. \\
& \left.+\frac{1}{\exp \left(\beta\left(-\varepsilon+\hbar \omega_{q}\right)\right)+I}\right] \delta\left(\hbar \omega_{q}-\varepsilon-\varepsilon^{\prime}\right)
\end{aligned}
$$

Here $q$ is the magnitude of the phonon wave vector and $\hbar \omega_{q}$ is the phonon energy. The overall factor of $q$ arises from an assumption that the squared matrix element for the interaction is proportional to $q$ and the $\delta$ function assures energy conservation. The total scattering rate is an average of $\Gamma(\varepsilon, q)$ over $\varepsilon$ and $q$. The weighting function for the energy average is the derivative of the Fermi function $f^{\prime}(\varepsilon)$ and the average over phonons must be consistent with energy conservation. This leads to the following sequence for the phonon integrals

$$
\int d^{3} q \rightarrow \underset{\text { Fermi }}{\int d_{\text {Surface }}^{3} q} \cong 2 \pi \int_{0}^{2 q_{F}} q d q
$$

We have implicitly assumed a spherical FS in this approximation. The results are adequate for copper without introducing another adjustable parameter to account for non-spherical FS shapes.

3. It has been previously pointed out that when phonons of wave vector $q$ satisfy the condition

$$
\frac{\mathrm{q}}{\mathrm{q}_{\mathrm{F}}} \ll \frac{\delta}{\mathrm{d}},
$$

where $\delta$ is the skin depth, $d$ the sample thickness, and $q_{F}$ is a phonon of wave vector equal to the Fermi surface 
$6 \cdot 874$
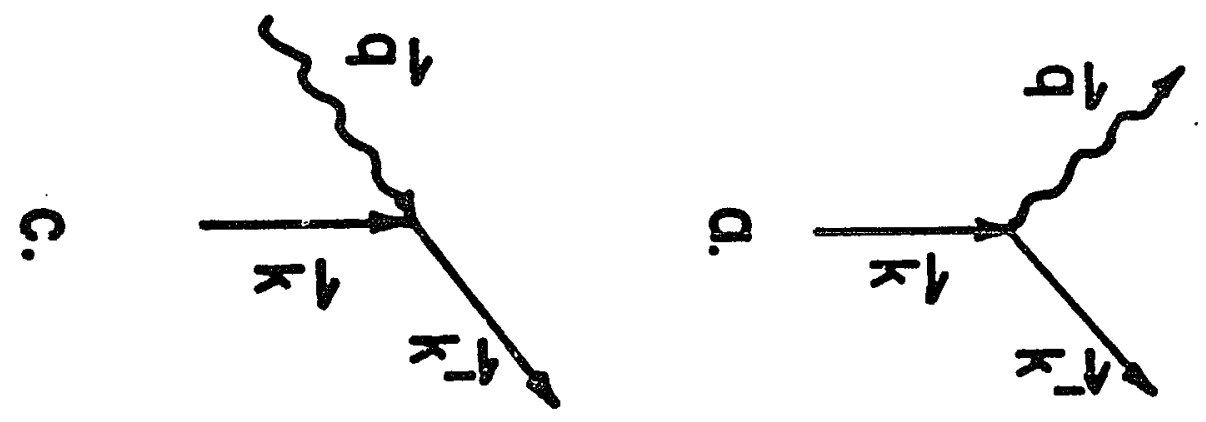

TIME

a
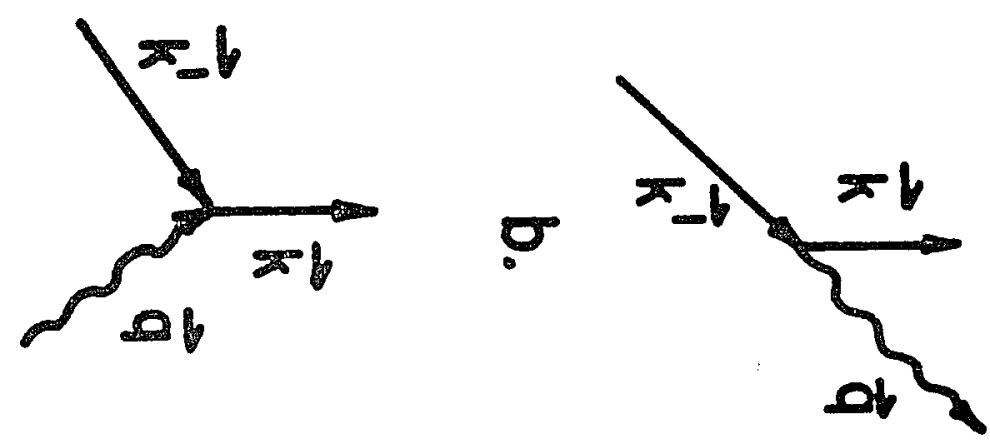
radius for the electron being. scattered, that these phonons will not remove an electron from an RFSE signal. Thus, when averaging the e-p matrix element over $q$, an effectiveness function, $\gamma(q)$, should be included. The appropriate function is derived in Section B. The functional form which is derived there is closely approximated by

$$
\gamma(q)=\tanh \left(\frac{\omega_{q} d}{\alpha}\right)
$$

and we use this form in the data fitting procedure. The parameter $\alpha$ is adjustable and is used to fit the experimental data.

4. Several years ago Gantmakher pointed out that phonons would be ineffective in scattering electrons when their wave vectors were larger than twice the $k$-space dimensions of the piece of FS on which an RFSE orbit was observed. This fact has also been discussed in connection with e-p scattering in the electrical resistivity of bismuth and antimony where the FS dimensions are small compared to phonon wave vector magnitudes. In copper this is not a predominate effect at lower $\left(1-10^{\circ} \mathrm{K}\right)$ temperatures, but in order to make the analysis procedure more complete, we allow for this possibility in our overall fitting procedure. The physical fact that e-p scattering has a cutoff when $q 22 k_{F}$ is manifest in the integral of phonons over $\Gamma(\varepsilon, q)$ having a noticeable temperature dependence when $2 k_{F} / q \approx 5$ or less. The upper limit of the phonon averaging integral is the ratio of the maximum phonon wave vector, 
$\mathrm{q}_{D^{\prime}}$ to the average $\mathrm{q}$ at the temperature of interest. In temperature units the upper limit is $\theta_{D} / T$, where $\theta_{D}$ is the Debye temperature. The effect of the cutoff for small pieces of Fermi surface is to cause the effective $\theta_{D}$, $\theta_{D}^{*}$ to be much smaller than that corresponding to the maximum phonon energy. A complete discussion of these considerations is given by Tsai. ${ }^{32}$ We use $\theta_{D}$ in the upper limit of integration for copper since the cutoff condition $q \geq 2 k_{F}$ is not approached in this case.

The effectiveness parameter, $\alpha$, has a simple physical interpretation due to Eq. (4). Rewrite Eq. (4) as

$$
\frac{\mathrm{gd}}{\mathrm{q}_{\mathrm{F}} \delta}=\frac{\omega_{\mathrm{g}} \mathrm{d}}{\omega_{\mathrm{q}_{F}} \delta}=\frac{\omega_{\mathrm{q}} \mathrm{d}}{{ }_{\mathrm{D}}^{*} \delta}>1 \text {. }
$$

Phonons become effective when

$$
\frac{\omega_{q} d}{\alpha}>1 \text {. }
$$

Thus, $\alpha$ should be of order $\theta_{D}^{*} \delta$. This shows that if $\theta_{D}^{*}$ is low the scattering out of an RFSE orbit becomes effective at lower temperatures than for larger values of $\theta_{D}^{*}$. That is, for small pieces of FS the scattering is effective at lower temperatures than for larger pieces of FS.

5. In order to compare the e-p scattering rate $\Gamma(\varepsilon, q)$ with experimental data an average over phonons which is weighted by the effectiveness function must be taken. Thus,

$$
\overline{\Gamma(\varepsilon)}=\int_{0}^{q_{\max }} \Gamma(\varepsilon, q) \gamma(q) d^{3} q .
$$


This effective scattering rate can be most easily calculated by making the change of variables $y=\beta \omega_{q}$ and $x=\beta \varepsilon$. Then $\Gamma(\varepsilon)$ is proportional to $T^{3} H(x)$, given by $T^{3} H(x)=T^{3} \int_{0}^{\theta * / T} \tanh \left(\frac{T y d}{\alpha}\right) y^{2}\left[\frac{2}{e^{y}-1}+\frac{1}{e^{x+y}+1}+\frac{1}{e^{-x+y}+1}\right] d y$.

There are two adjustable parameters in our formation so far: $\theta_{D}^{*}$ and $\alpha$. For large values of $\theta_{D}$ the integral is insensitive to the magnitude of $\theta_{D}^{*}$ and $\theta_{D}$ is a reasonable value to use. When $\theta_{D}^{*}$ is of order five times or less the temperature range over which data are obtained then its precise value becomes an important factor in a data-fitting procedure. The numerical value of $\alpha$ is determined by the lowest temperature data of $\ln A$ vs a function of $T$.

6. In the final averaging process an average of electrons of different energies near the Fermi energy must be performed. The probability that a given electron will traverse a sample of thickness a is given by

$$
\mathrm{P}=\mathrm{z}^{\mathrm{d}} \mathrm{e}^{-\mathrm{GdT} \mathrm{T}^{3} \mathrm{H}(\mathrm{x})}
$$

It is $\mathrm{P}$, not $\mathrm{H}(\mathrm{x})$ which must be averaged over different electrons. Using the symmetry $H(x)=H(-x)$ and the identity for the derivative of the Fermi function

$$
f^{\prime}(x)=\frac{1}{4 \cosh ^{2}(x / 2)}
$$

the integral to be performed is

$$
A_{1}=\frac{A_{0}}{2} \int_{0}^{\infty} \frac{z^{d} \exp \left(-G d T^{3} H(x)\right)}{\cosh ^{2}(x / 2)} d x
$$


This is the single pass formula for the RFSE amplitude, where $G$ is the e-p scattering rate per ${ }^{\circ} K^{3}$.

7. The quantity $A_{1}$ is not the total RFSE amplitude because it may be possible that electrons will traverse the sample thickness more than once. Define the amplitude for $\mathrm{N}$ traverses to be:

$$
A_{N}=\frac{A_{0}}{2} \int_{0}^{\infty} \frac{\exp \left(-G_{N A T}{ }^{3}(x)\right)}{\cosh ^{2}(x / 2)} z^{N d} d x,
$$

then the measured signal amplitude will be

$$
A=\sum_{N=1}^{\infty} A_{N} \text {. }
$$

The expression for $\mathrm{A}_{\mathrm{N}} / \mathrm{A}_{\mathrm{O}}$ contains four adjustable parameters: $G$, the electron-phonon scattering strength; $\alpha$, the effectiveness parameter; $\theta_{D}^{*}$, the effective Debye temperature; and $\nu_{I}$, the impurity scattering frequency.

8. In KAG it was pointed out that the exact expression for A, Eq. (1) of KAG, showed curvature when InA was plotted against $\mathrm{T}^{3}$. So does Eq. (13). This deviation from a $\mathrm{T}^{3}$ law increases as sample thickness decreases and is only appreciable at the high temperature end of measurement ranges. Empirically we have found that the addition of a scattering frequency proportional to $\mathrm{T}^{5}$ and $\sqrt{\mathrm{d}}$ gives the required correction to the multiple pass expression, Eq. (13), and causes the experimentally observed $\mathrm{T}^{3}$ behavior to be predicted. In what follows we give a simple justification of why this type of behavior might be expected. 
In making an RFSE measurement, one in general applies an electric field, $E_{0}$, in the skin depth of a sample and monitors the changes in $E_{0}$ due to the current produced in the skin depth to cancel it. The solution to this problem involves a very complicated surface impedance calculation which has been worked on by many authors over the past few years. The $\sqrt{d} \mathrm{~T}^{5}$ dependence can be roughly obtained by assuming that the induced current in the skin depth satisfies Newton's law with a damping term. That is,

$$
\frac{d J}{d t}=\xi E_{0}-\frac{J}{\tau}
$$

where $\xi$ can only be determined from a complete impedance calculation and $\tau$ is a relaxation time for the surface current which, because it is a bulk property, is proportional to $\mathrm{T}^{-5}$. During an RFSE resonance the surface currents are altered somewhat because the orbiting electrons which just span the sample thickness cause a current flow, $J_{0}$, whether or not $E_{O}$ is present. This current exists over a distance $x_{0}$ in the skin depth as shown in Fig. 10. Since the RFSE measurement conditions are such that the rf electric field appears static to the orbiting electrons $\left(\omega_{\text {rf }} \ll \Omega\right)$, the relevant time in which a resonant set of electrons would be involved in the surface current is the time spent in the skin depth. If $v$ is the velocity of electrons on the resonant orbit, then $t=x_{0} / v$ is the time of importance. The limits of integration for $\mathrm{Eq}$. (14) are $J=J_{0}$ at $t=0$ and $J=J(t)$ at $t=t$, and the result of 


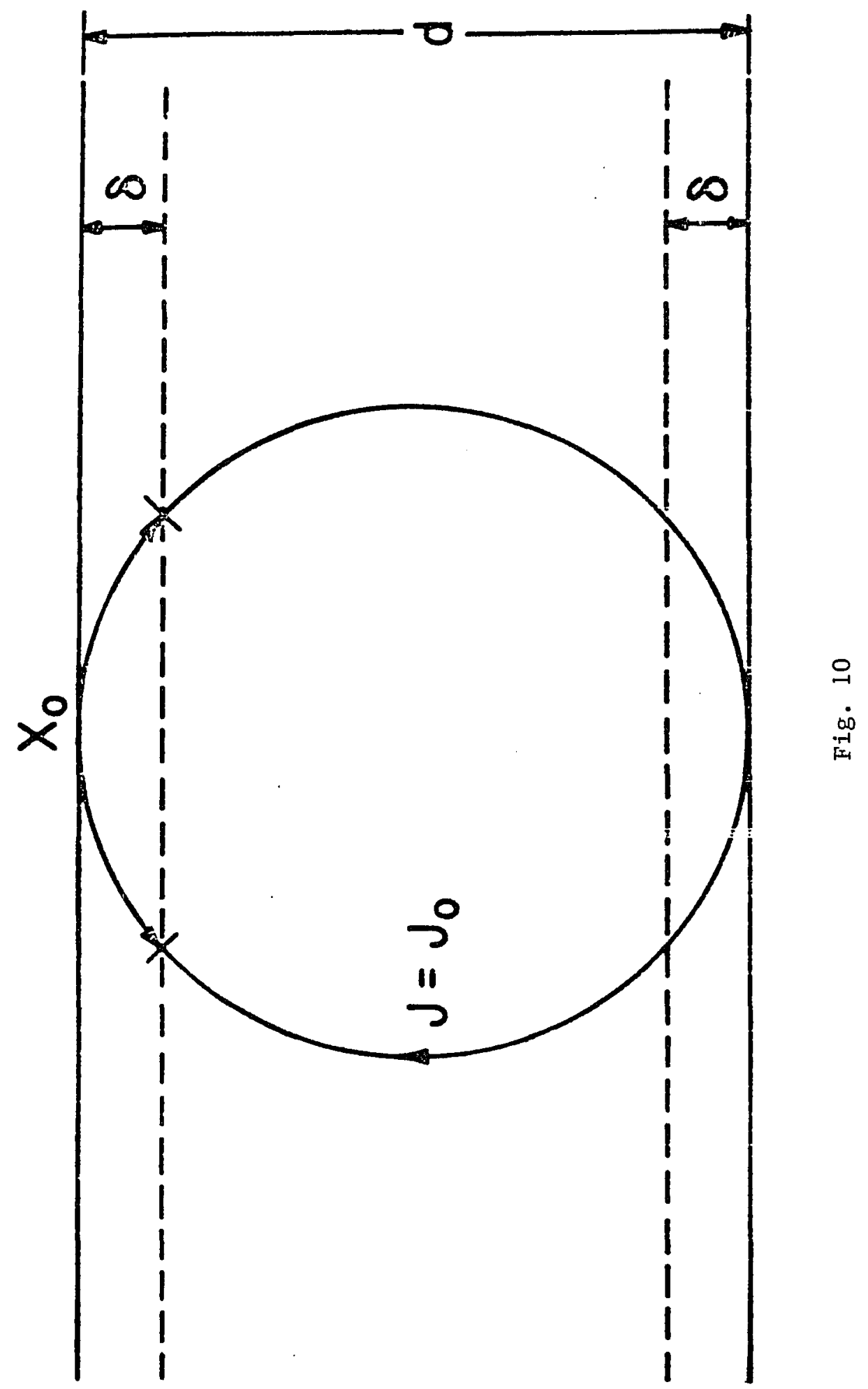


integration is

$$
J(t)=\xi_{0} E_{0}+\left(J_{0}-\xi_{0} \tau\right) e^{-t / \tau} .
$$

The interesting part of this equation for the RFSE is that part proportional to $J_{0^{\prime}}$ or that is the portion contributing to the RFSE amplitude, $J_{R}$. Thus, the signal amplitude should in fact be proportional to $J_{R}=J_{0} \exp (-t / \tau)$ where $J_{0}$ is proportional to Eq. (11). From Fig. 10 it can be seen that $x_{0} \simeq 2 \mathrm{~d} \sqrt{\delta / d} \propto \sqrt{\mathrm{d}}$. Now, since $1 / \tau \propto \mathrm{T}^{5}$ and $t=x_{0} / v \propto \sqrt{d}$, these considerations predict that $\ln A$ contain an additional term proportional to $\mathrm{T}^{5} \sqrt{\mathrm{d}}$. Much more sophisticated considerations are required to obtain the magnitude of this term and we include the magnitude as an adjustable parameter.

This concludes the factors included in our model. All of these factors can be summarized as follows

$$
A=A_{0}\left(\sum_{N=1}^{\infty} A_{N}\right) e^{-Q T^{5} \sqrt{d}}
$$

or

$$
\ln A / A_{0}=\ln (E q \cdot(13))-Q T^{5} \sqrt{d} .
$$

The final fit to the data, then requires five adjustable parameters including $Q$, only four of which are totally independent (see Eqs. 6 or 7). In practice it is possible to either measure or calculate $\theta_{D}^{*}$ from other considerations so only four parameters, $\nu_{I}, G, \alpha$, and $Q$, will in general be determined from fits of this theory to experimental data. 


\section{B. Scattering Effectiveness Calculation}

In this section a model calculation for $\gamma(q)$, the probability that a phonon of momentum $q$ scatters an electron out of an RFSE orbit, is given.

We presume the sample is homogeneous and isotropic with smooth parallel faces separated by a distance d. The radio frequency electric field penetrates both surfaces a skin depth $\delta$ where $\delta \ll$ d. Additionally we assume the radius of the electronic orbits (recall the external magnetic field is parallel to the sample surfaces) most effective in the resonance process to be

$$
r=\frac{1}{2}(d-\delta)
$$

Let $\Delta$ represent a spatial translation of the circular resonance orbit in a direction perpendicular to the sample surfaces. It can be shown that a spatial translation $\Delta$ leads to a new trajectory intersecting a surface of the sample with probability

$$
P(\Delta)= \begin{cases}\frac{\Delta}{\delta} & \text { if } \Delta<\delta \\ 1 & \text { if } \Delta>\delta .\end{cases}
$$

Kinematic considerations for the electron-phonon scattering will determine how the spatial translation occurring as a consequence of such an event is a function of the initial electron momentum $\vec{k}$ and the phonon momentum $\vec{q}$. Here $\mathrm{q} \ll \mathrm{k}$ and only the direction of the electron momentum is significantly altered by the phonon absorption or emission as described below. 
If one assumes that the Fermi surface of the material is spherical, then the initial and final electron momenta, $\vec{k}$ and $\vec{k}$ ' respectively, will be of essentially equal magnitude. Furthermore, $\vec{k}$ and $\vec{q}$ will essentially be at right angles. Because of this only phonons approaching or leaving the electron in a plane perpendicular to $\vec{k}$ may scatter the electron; other phonons would violate the energy conservation requirement.

The electron momentum is always perpendicular to the radius vector of the circular resonance orbit and in this way, given $r$, the direction of the electron momentum defines the center of the orbit. Phonon absorption or emission yields a new direction for the electron momentum and a new orbit center is consequently established. For an illustration of this point and as a guide to what follows see Fig. 11. A new orbit center corresponds to a translation $\Delta$ of the orbit towards or away from a sample surface. The direction of $\vec{q}$ will be parameterized by two angles $\theta$ and $\phi$ in a way that conforms to our remarks in the previous paragraph. Our subsequent derivation ignores the role of the $x$-component of $\vec{q}$ in the scattering from resonance. We expect this to be non-negligible only for large $q$.

All of the preceding assumptions are incorporated into the diagram shown in Fig. 11 and from this it is seen that $\Delta \approx x|\cos \theta|=\frac{r q}{k}|\cos \phi \cos \theta| \approx \frac{q \delta}{q_{c r i t}}|\cos \phi \cos \theta|$ where a critical phonon momentum

$$
q_{\text {crit }}=\frac{2 \mathrm{k} \delta}{\mathrm{d}}
$$




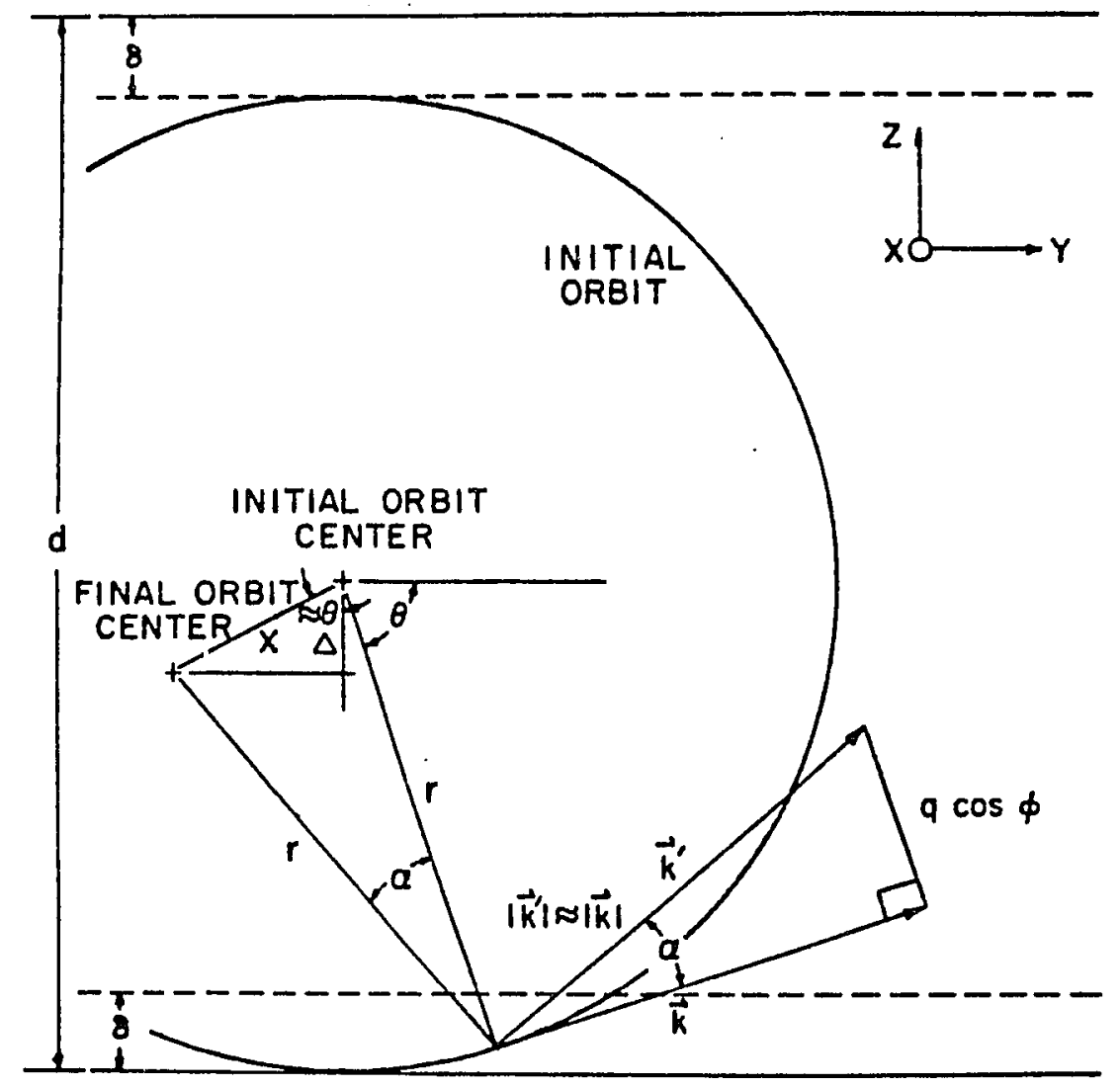

Fig. 11 
has been introduced. In terms of these parameters the probability of an orbit intersecting a surface becomes

$$
P(\vec{q})=\left\{\begin{array}{cl}
\frac{q}{q_{\text {crit }}}|\cos \phi \cos \theta|, & \text { if } \frac{q \delta}{q_{\operatorname{crit}}}|\cos \phi \cos \theta|<1 \\
1 & , \text { if } \frac{q \delta}{q_{\text {crit }}}|\cos \phi \cos \theta|>1
\end{array}\right.
$$

The total scattering probability is obtained by integration over the orbit as follows:

$$
\gamma(q)=\frac{\int_{0}^{2 \pi} P(\vec{q}) d \phi d \theta}{\int_{0}^{2 \pi} \int d \phi d \theta}
$$

This integration, in general, must be done numerically because the analytic form for $P(\vec{q})$ depends on an inequality involving $q$, $\phi$ and $\theta$. However, in the case where $q \leq q_{\text {crit' }}$, the integration can be done analytically and we find

$$
\gamma(q)=\frac{4}{\pi^{2}} \frac{q}{q_{\text {crit }}} \quad \text { for } q \leq q_{\text {crit }} \text {. }
$$

The result of numerical integration for $\gamma(q)$ out to $q / q_{\text {crit }}=10.0$ is shown in Fig. 12. In addition a plot of the hyperbolic tangent of $0.3 \mathrm{x}$ is given to show its similarity to $\gamma(q)$ and justify its use in our numerical fitting procedure. An approximation used in obtaining $\gamma(q)$ causes the curve to underestimate the scattering probability at large values of $q$. This is because the $x$ component of $\vec{q}$ for large $|\vec{q}|$ will become effective in scattering electrons from resonance. Since this mechanism was not included in the model, the hyperbolic tangent function used to represent $\gamma(q)$ in the fitting procedure is justifiable because it gives slightly larger values of the scattering probability 


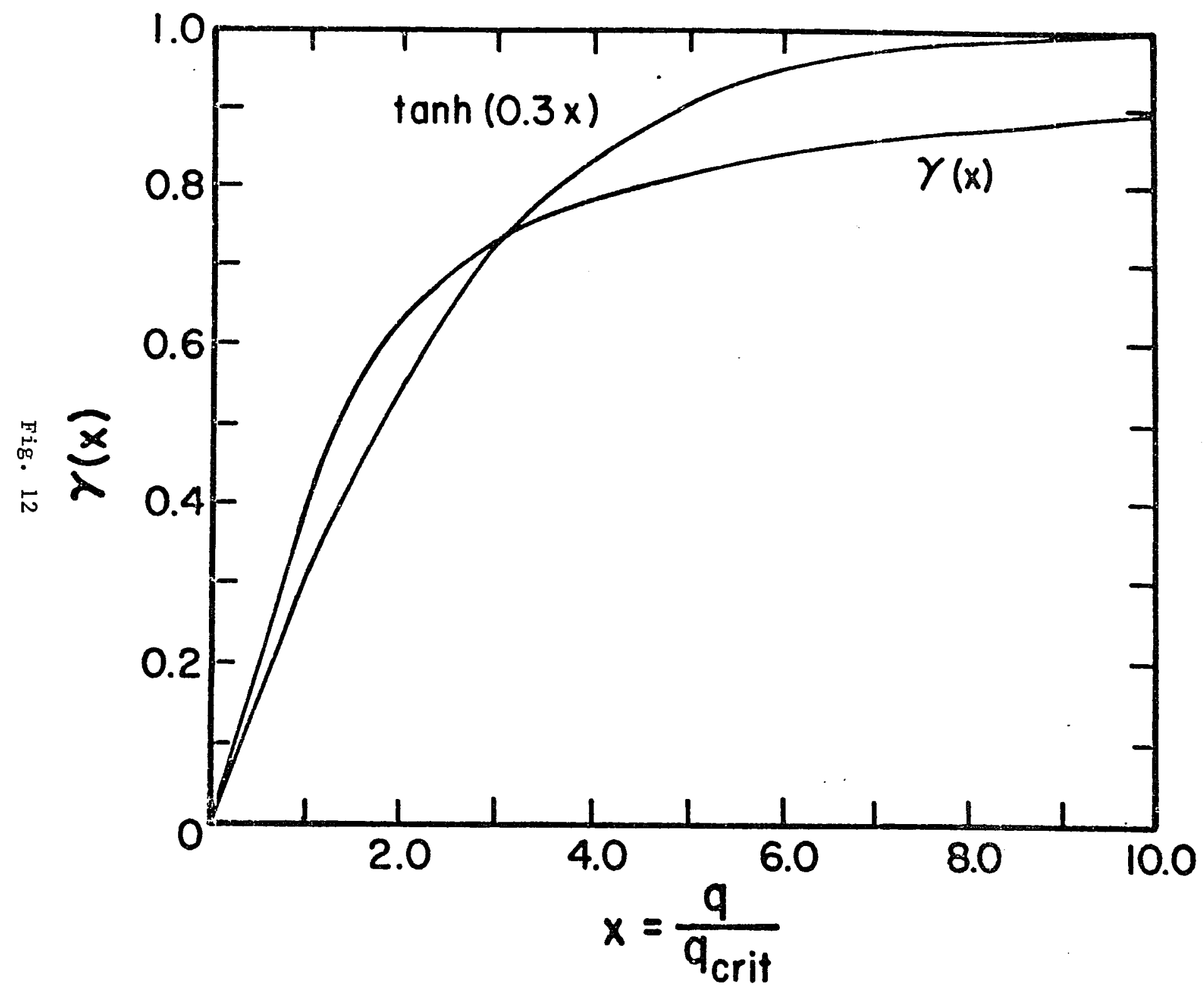


than does $\gamma(q)$ at large $q$ but has qualitatively the same shape. 


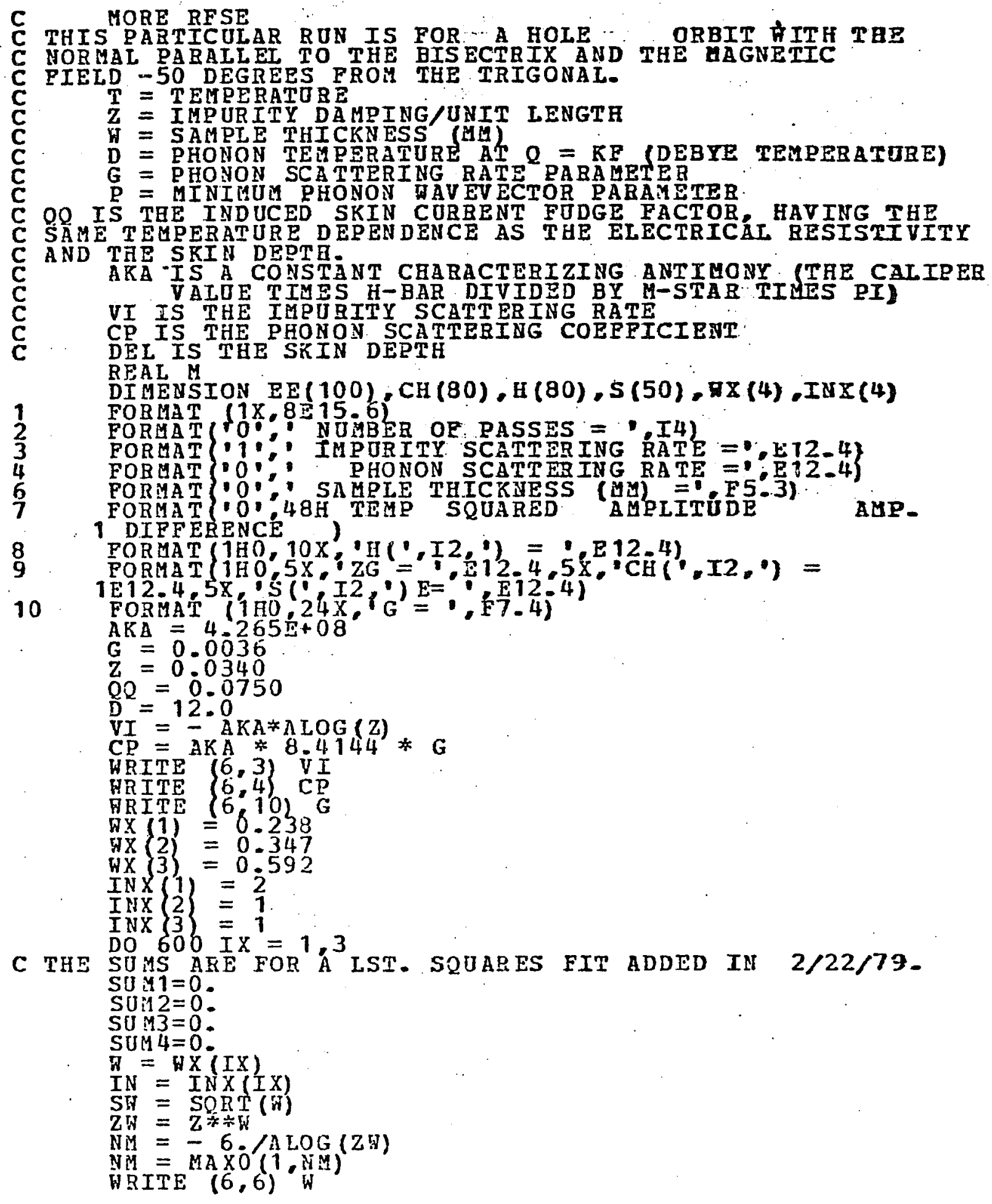




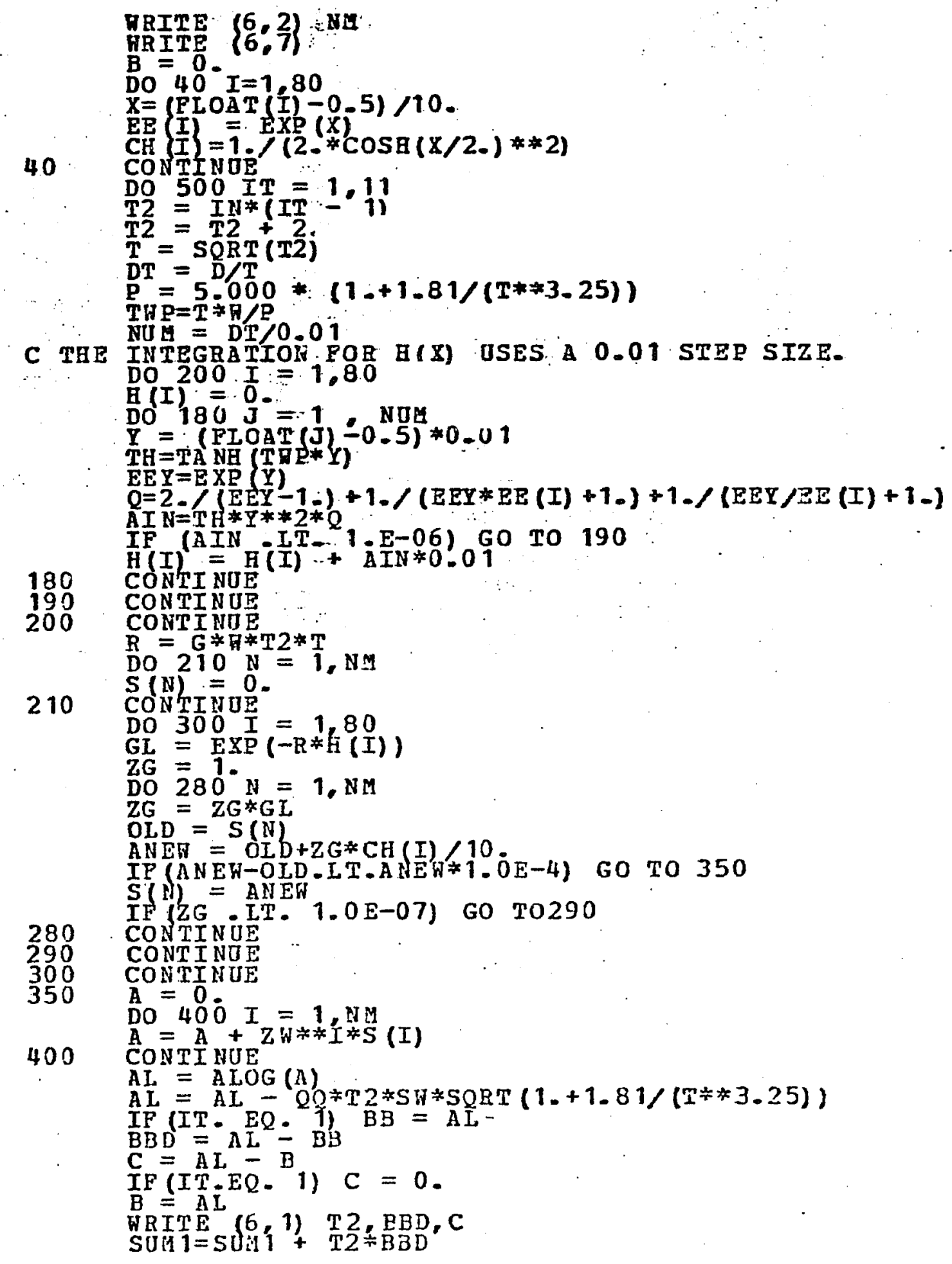




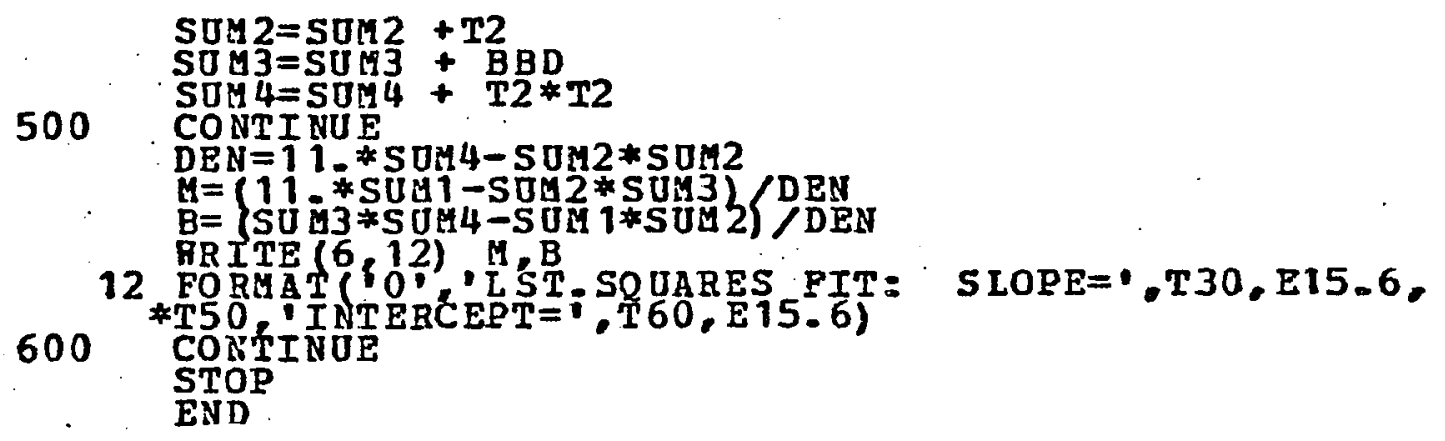


VITA

Thomas C. O'Hara was born in Ft. Worth, Texas, on May 15, 1947. His secondary education was completed at Nolan High School in Ft. Worth, and he received a Bachelor of Science degree in physics in 1969 from the University of Texas at Austin. The following year was spent in graduate work in physics at Texas Tech University. The next eighteen months were spent in the U.S. Army. In the spring of 1972 he enrolled for graduate work in physics at Louisiana State University and received a Master of Science degree in December of 1973. In May of 1970 he was married to the former Joy Lee Smith, and they have two children. He is a member of the American Association of Physics Teachers and has been elected to Sigma Pi Sigma and Phi Kappa Phi honor societies. He is presently a candidate for the degree of Doctor of Philosophy. 


\section{EXAMINATION AND THESIS REPORT}

Candidate: Thomas C. O'Hara

Major Field: Physics

Title of Thesis: Electron-Phonon Scattering Rates in Antimony: Radio-Frequency Size Effect

Approved:

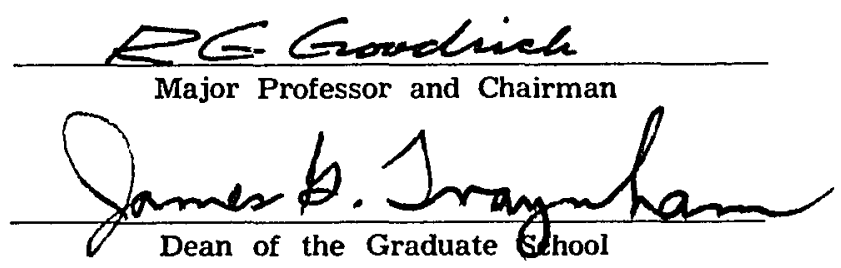

EXAMINING COMMITTEE:

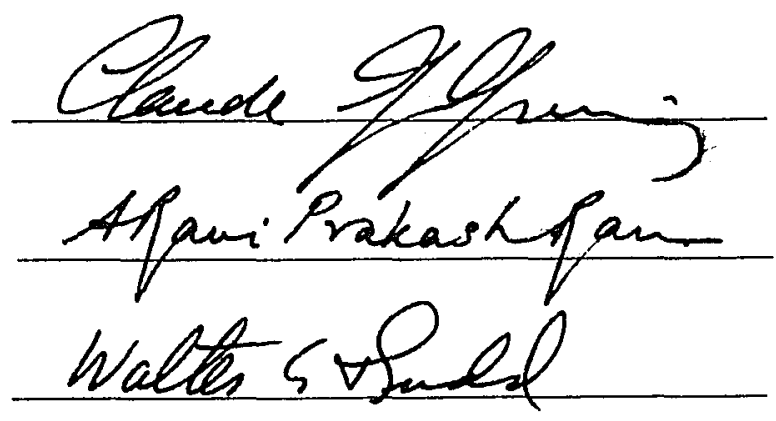

Rolut D. Itroseg

Date of Examination: 